\title{
Dense Gas Towards the RX J1713.7-3946 Supernova Remnant
}

\author{
Nigel I. Maxted ${ }^{1,6}$, Gavin P. Rowell ${ }^{1}$, Bruce R. Dawson ${ }^{1}$, Michael G. Burton ${ }^{2}$, Yasuo Fukui ${ }^{3}$, \\ Jasmina Lazendic ${ }^{4}$, Akiko Kawamura ${ }^{3}$, Hirotaka Horachi ${ }^{3}$, Hidetoshi Sano ${ }^{3}$, Andrew J. Walsh ${ }^{5}$, \\ Satoshi Yoshiike ${ }^{3}$ and Tatsuya Fukuda ${ }^{3}$ \\ ${ }^{1}$ School of Chemistry \& Physics, University of Adelaide, Adelaide, SA 5005, Australia \\ ${ }^{2}$ School of Physics, University of New South Wales, Sydney, NSW 2052, Australia \\ ${ }^{3}$ Department of Astrophysics, Nagoya University, Furocho, Chikusa-ku, Nagoya, Aichi 464-8602, Japan \\ ${ }^{4}$ School of Physics, Monash University, Melbourne, Victoria 3800, Australia \\ 5International Centre for Radio Astronomy Research, Curtin University, GPO Box U1987, Perth, Australia \\ ${ }^{6}$ Email: nigel.maxted@adelaide.edu.au
}

(Received July 16, 2013; ACCEPTED October 1, 2013)

\begin{abstract}
We present results from a Mopra $7 \mathrm{~mm}$-wavelength survey that targeted the dense gas-tracing CS(1-0) transition towards the young $\gamma$-ray-bright supernova remnant, RX J1713.7-3946 (SNR G 347.3-0.5). In a hadronic $\gamma$-ray emission scenario, where cosmic ray $(\mathrm{CR})$ protons interact with gas to produce the observed $\gamma$-ray emission, the mass of potential $\mathrm{CR}$ target material is an important factor. We summarise newly discovered dense gas components, towards Cores $\mathrm{G}$ and L, and Clumps N1, N2, N3, and T1, which have masses of $1-10^{4} \mathrm{M}_{\odot}$. We argue that these components are not likely to contribute significantly to $\gamma$-ray emission in a hadronic $\gamma$-ray emission scenario. This would be the case if RX J1713.7-3946 were at either the currently favoured distance of $\sim 1 \mathrm{kpc}$ or an alternate distance (as suggested in some previous studies) of $\sim 6 \mathrm{kpc}$.

This survey also targeted the shock-tracing $\mathrm{SiO}$ molecule. Although no $\mathrm{SiO}$ emission corresponding to the RX J1713.7-3946 shock was observed, vibrationally excited SiO(1-0) maser emission was discovered towards what may be an evolved star. Observations taken $1 \mathrm{yr}$ apart confirmed a transient nature, since the intensity, line-width, and central velocity of $\mathrm{SiO}(\mathrm{J}=1-0, \mathrm{v}=1,2)$ emission varied significantly.
\end{abstract}

Keywords: cosmic rays - gamma rays: ISM - ISM: clouds - molecular data - supernovae: individual: RX J1713.7-3946

\section{INTRODUCTION}

RX J1713.7-3946 (G 347.3-0.5) is a supernova remnant (SNR) that is bright in X-ray emission (Pfeffermann et al. 1996; Cassam-Chenai et al. 2004; Acero et al. 2009) and is one of the brightest sources in the TeV $\gamma$-ray sky (Aharonian et al. 2006, 2007). This remnant is therefore ideal for investigating the possibility of acceleration of cosmic rays (CRs) in the shocks of SNRs.

Knowledge of the distribution of matter towards RX J1713.7-3946 is important to help distinguish between models of $\gamma$-ray emission that are dominated by high energy electrons (inverse-Compton scattering of photons) and models dominated by CR hadrons ( $p-p$ interactions). The latter scenario requires target material for CRs. CR target material may be in any chemical form, including molecular and atomic gas, which have been studied with CO (Fukui et al.
2003; Moriguchi et al. 2005; Fukui 2008) and HI (+CO) (Fukui et al. 2012), respectively.

We take particular interest in the study of dense molecular gas, which is well traced by CS(1-0) emission (critical density $\sim 10^{5} \mathrm{~cm}^{-3}$ ). This transition highlights gas mass possibly missed by other tracers (such as CO) and aids in the prediction of possible small-scale $\gamma$-ray emission features caused by energy-dependent CR diffusion into dense gas (e.g. Gabici, Aharonian, \& Casanova 2009; Casanova et al. 2010; Fukui et al. 2012; Maxted et al. 2012a).

The latest Fermi-LAT observations (Abdo et al. 2011) towards RX J1713.7-3946 exhibit a low but hard-spectrum flux of 1-10 GeV $\gamma$-ray emission uncharacteristic of previous hadronic models (Porter, Moskalenko, \& Strong 2006; Aharonian et al. 2007; Berezhko \& Volk 2010; Ellison et al. 2010; Zirakashvili \& Aharonian 2010). However, if one considers an inhomogeneous ISM into which the SNR shock has 


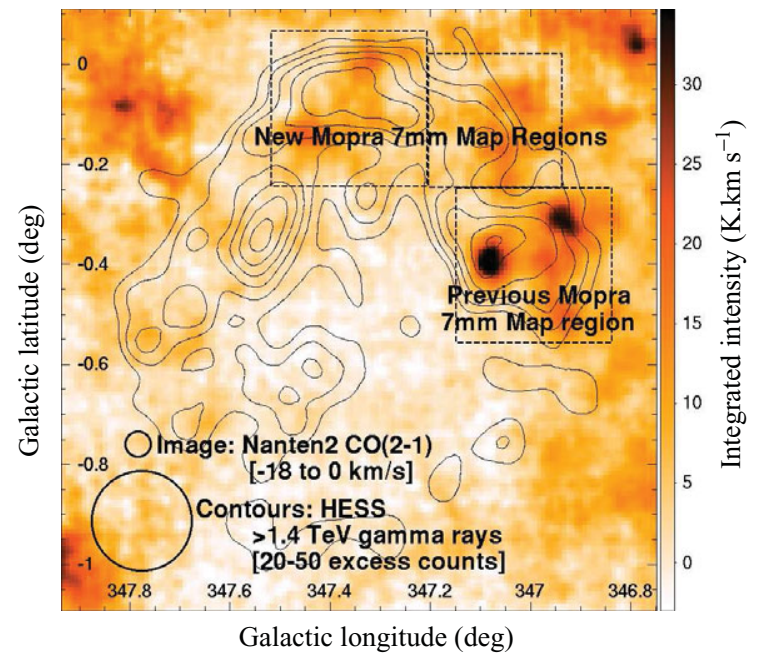

Figure 1. Integrated Nanten2 $\mathrm{CO}(2-1)$ emission image (Fukui 2008) of gas believed to be associated with RX J1713.7-3946 $\left(\mathrm{v}_{\mathrm{LSR}}=-18\right.$ to $0 \mathrm{~km} \mathrm{~s}^{-1}$ ) with overlaid HESS $>1.4 \mathrm{TeV} \gamma$-ray photon excess count contours (Aharonian et al. 2007). Three square regions (one, the southern-most, from a previous investigation) indicate the extent of Mopra $7 \mathrm{~mm}$ mapping carried out in this study.

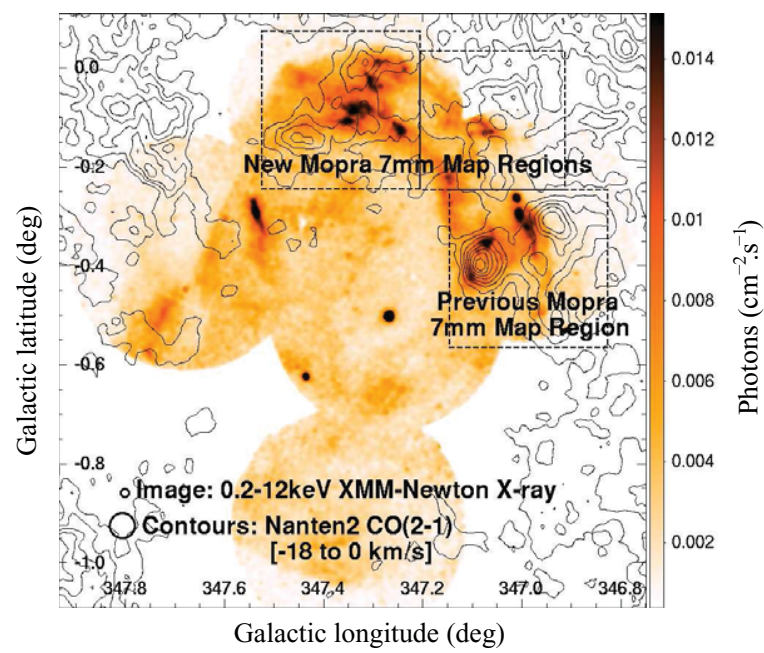

Figure 2. An XMM-Newton 0.5-4.5 keV X-ray image (Acero et al. 2009) with overlaid integrated Nanten $2 \mathrm{CO}(\mathrm{J}=2-1)$ emission $\left(\mathrm{v}_{\mathrm{LSR}}=-18\right.$ to $0 \mathrm{~km} \mathrm{~s}^{-1}$ ) contours. $\mathrm{CO}(\mathrm{J}=2-1)$ contours span $5 \mathrm{~K} \mathrm{~km} \mathrm{~s}^{-1}$ to $40 \mathrm{~K} \mathrm{~km} \mathrm{~s}^{-1}$ in increments of $5 \mathrm{~K} \mathrm{~km} \mathrm{~s}^{-1}$. The XMM-Newton image has been exposurecorrected and smoothed with a Gaussian of FWHM $=30$ arcsec. Three square regions (one, the southern-most, from a previous investigation) indicate the extent of Mopra $7 \mathrm{~mm}$ mapping carried out in this study.

expanded, the existence of a hadronic component may still be plausible (Zirakashvili \& Aharonian 2010; Inoue et al. 2012). There is also support for such a scenario when considering the existence of additional atomic gas together with the molecular (Fukui et al. 2012).

RX J1713.7-3946 exhibits a shell-like structure at keV $\mathrm{X}$-ray and $\mathrm{TeV} \gamma$-ray energies (see Figures 1 and 2), with the
$\mathrm{keV} \mathrm{X}$-ray emission best corresponding to a void in molecular gas, bordering molecular cores at a kinematic distance of $\sim 1 \mathrm{kpc}\left(\mathrm{v}_{\mathrm{LSR}} \sim-10 \mathrm{~km} \mathrm{~s}^{-1}\right.$ ) (Fukui et al. 2003; Moriguchi et al. 2005). This distance is consistent with $\mathrm{X}$-ray absorption modelling by Pfeffermann et al. (1996) for a plausible SNRage ( $\sim 1600$ yrs old) that corresponds to a progenitor event observed by Chinese astronomers in $393 \mathrm{AD}$ (Wang, Qu, \& Chen 1997).

Fessen et al. (2012) argue that if a SN 393 AD connection to RX J1713.7-3946 is assumed, the initial RX J1713.7-3946 SN explosion must have been optically subluminous for the $\sim 1 \mathrm{kpc}$ distance to hold. Irrespective of this, recent work still favours a $\sim 1 \mathrm{kpc}$ distance (e.g. Fukui et al. 2012; Inoue et al. 2012), and an examination of $\mathrm{keV}$ $\mathrm{X}$-ray emission (Figure 2) suggests some degree of anticorrelation with $\mathrm{CO}(2-1)$ peaks at $\mathrm{v}_{\mathrm{LSR}} \sim-10 \mathrm{~km} \mathrm{~s}^{-1}$ (a kinematic distance of $\sim 1 \mathrm{kpc}$ ). Sano et al. (2010) note that peaks of synchrotron radiation on the boundary of the molecular clump, Core $\mathrm{C}$, may suggest a compression triggered by the RX J1713.7-3946 shock. Similarly, the edge of Core D is coincident with an X-ray intensity peak, consistent with shock compression at a distance of $\sim 1 \mathrm{kpc}$. Furthermore, the northern and western regions of RX J1713.7-3946, where cores D and $\mathrm{C}$ are located, correspond to peak fluxes of $\mathrm{TeV} \gamma$-ray emission. Good gas- $\gamma$-ray overlap, like that seen towards RX J1713.7-3946 and the $\sim 1 \mathrm{kpc}$ gas, is indeed expected in a hadronic scenario for $\gamma$-ray emission.

In a previous study (Maxted et al. 2012a), we surveyed an $18 \times 18$ arcmin region centred on $[l, b]=[346.99,-0.41]$ (southern-most region indicated in Figure 1), and found dense gas associated with molecular cores A, C, and D (see Figure 3). In this study we extend the dense gas survey with another two mapped regions, to encompass Core D and the northern peak in $\mathrm{TeV} \gamma$-ray emission, and several bright $\mathrm{keV} \mathrm{X}$-ray emission features. CS(1-0) emission can probe the dense gas towards RX J1713.7-3946, which, as highlighted earlier, may be important in a hadronic scenario. The Mopra radio telescope spectrometer is capable of simultaneously recording $\mathrm{CS}(1-0)$ emission, while observing the shock-tracing transition $\mathrm{SiO}(1-0)$. This allows us to further test the $\sim 1$ kpc RX J1713.7-3946 kinematic distance solution through attempting to trace the SNR shock, while undertaking our survey of dense gas. The shock-tracing molecule, $\mathrm{SiO}$, has been observed towards shocked gas associated with other (albeit older) SNRs (e.g. Ziurys, Snell, \& Dickman 1989; Nicholas et al. 2012), so may possibly be present towards gas associated with RX J1713.7-3946.

We also note the usefulness of other molecular lines simultaneously observed at $7 \mathrm{~mm}$ with the Mopra telescope. These include emission from isotopologues of CS, which are useful for probing optically thick regions, the vibrationally-excited modes of the $\mathrm{SiO}(1-0)$ rotational transition $(\mathrm{v}=1,2,3)$, which can sometimes be emitted in association with star formation or evolved stars, and the $\mathrm{CH}_{3} \mathrm{OH}(7(0)-6(1))$ transition that can highlight warm regions where $\mathrm{CH}_{3} \mathrm{OH}$ (methanol) is evaporated from dust grains. 

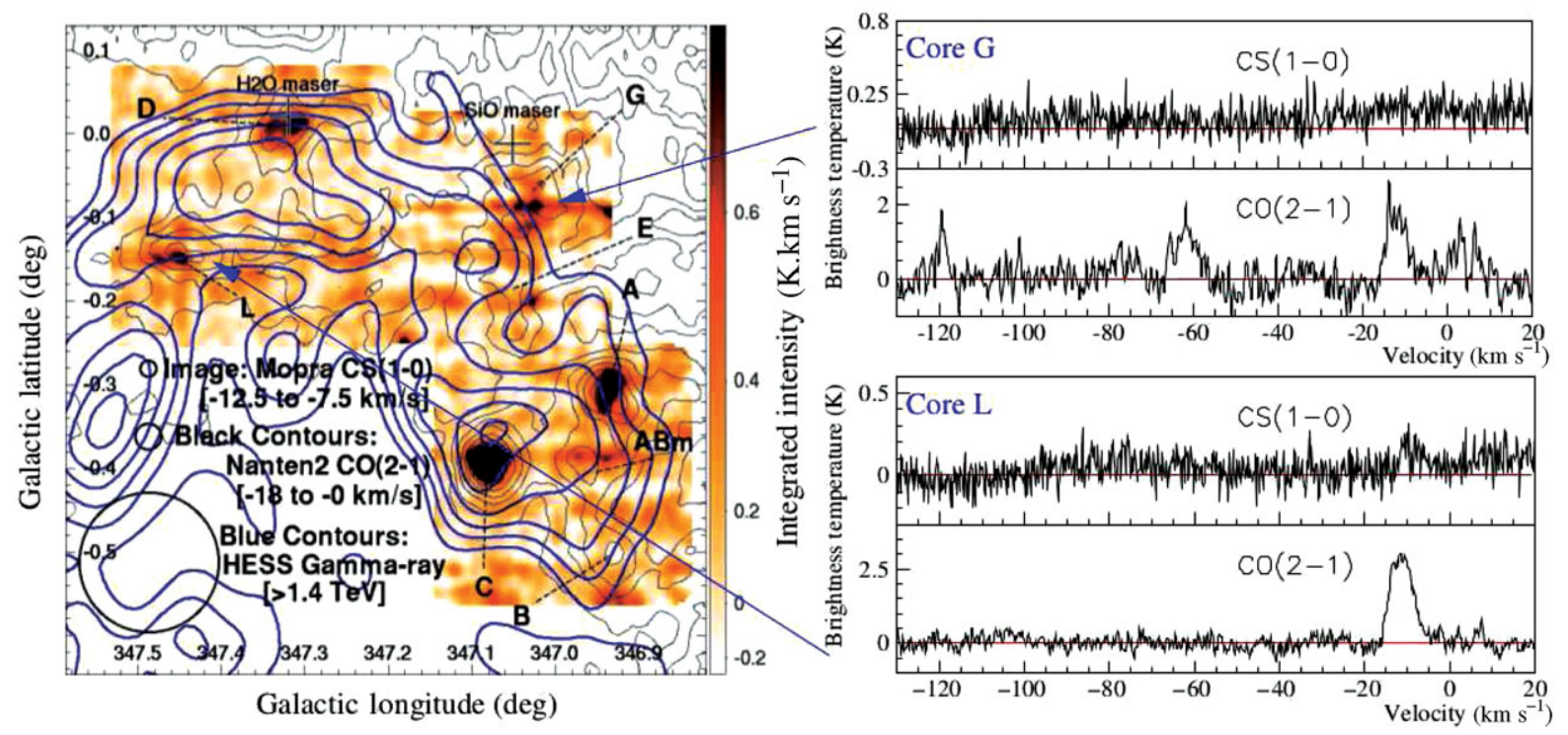

Figure 3. Left panel: Colour image of integrated CS(1-0) emission $\left(\mathrm{v}_{\mathrm{LSR}}=-12.5\right.$ to $\left.-7.5 \mathrm{~km} \mathrm{~s}^{-1}\right)$ from Mopra overlaid with black contours of $\mathrm{CO}(2-1)$ emission $\left(\mathrm{v}_{\mathrm{LSR}}=-18\right.$ to $0 \mathrm{~km} \mathrm{~s}^{-1}$ ) from Nanten2 (Fukui 2008), as well as solid blue contours of HESS $>1.4 \mathrm{TeV}$ excess emission (same levels as for Figure 1). Core names and the position of masers are indicated. $\mathrm{CO}(2-1)$ emission contour-levels are 5, 10, 15, 20, 25, 30, 35 , and $40 \mathrm{~K} \mathrm{~km} \mathrm{~s}^{-1}$. Right panel: We also show spectral profiles of molecular emission towards locations of interest (indicated).

\section{OBSERVATIONS}

In 2011 April, we recorded and co-added six Mopra OTF (on the fly) $19 \times 19$ arcmin area maps centred on $[l, b]=$ [347.36, -0.09], and five Mopra OTF $16 \times 16$ arcmin area maps centred on $[l, b]=[347.07,-0.11]$, to produce a data cube with two spatial (long/lat) and one spectral (velocity) dimension at $7 \mathrm{~mm}$ wavelengths. These maps were added to our map of an $18 \times 18$ arcmin region centred on $[l, b]=$ [346.991, -0.408], from our previous investigation (Maxted et al. 2012a).

For all our Mopra mapping data, the cycle time is $2.0 \mathrm{~s}$ and the spacing between scan rows is 26 arcsec. The velocity resolution of the $7 \mathrm{~mm}$ zoom-mode data is $\sim 0.2 \mathrm{kms}^{-1}$. The beam FWHM and the pointing accuracy of Mopra at $7 \mathrm{~mm}$ are $59 \pm 2$ arcsec and $\sim 6$ arcsec, respectively. The Mopra spectrometer, MOPS, was employed and is capable of recording 16 tunable, 4096-channel (137.5 MHz) bands simultaneously when in 'zoom' mode, as used here. A list of measured frequency bands, targeted molecular transitions, and achieved $\mathrm{T}_{\text {RMS }}$ levels are shown in Table 1.

OTF-mapping and deep ON-OFF switched pointing data were reduced and analysed using the ATNF analysis programs, Livedata, Gridzilla, Kvis, Miriad, and ASAP ${ }^{1}$. We assumed the beam efficiencies presented in Urquhart et al. (2010) to convert antenna intensity into main-beam intensity ( 0.43 and 0.53 for point source $\mathrm{CS}(1-0)$ and $\mathrm{SiO}(1-0)$ emission, respectively).

In addition to mapping, two deep ON-OFF switched pointings were performed in response to a detection of $\mathrm{SiO}(\mathrm{J}=$ $1-0, v=2$ ) emission (see Section 4.2.1). The first of these

\footnotetext{
${ }^{1}$ See http://www.atnf.csiro.au/computing/software/
}

two pointings was interrupted after achieving a signal-noise ratio similar to mapping data, so a second pointing with a longer exposure-time was performed $12 \mathrm{~d}$ later.

\section{SPECTRAL LINE ANALYSIS}

We use the CS(1-0) analysis outlined in Maxted et al. (2012a), and we briefly summarise it here. Gaussian functions were first fitted to all CS(1-0) emission lines using a $\chi^{2}$ minimisation method. Generally one can estimate CS(1-0) optical depth by comparing the $C S(1-0)$ intensity to the $C^{34} S(1-0)$ intensity, while assuming an abundance ratio, but of the new positions that exhibited CS(1-0) emission, none had corresponding $\mathrm{C}^{34} \mathrm{~S}(1-0)$ detections. We constrain optical depth by placing an optically thin (optical depth, $\tau \rightarrow 0$ ) lower limit and a conservative upper limit derived using the $\mathrm{C}^{34} \mathrm{~S}(1-0)$ band $\mathrm{T}_{\mathrm{RMS}}$ as an upper limit on $\mathrm{C}^{34} \mathrm{~S}(1-0)$ intensity, and assuming a $[\mathrm{CS}] /[\mathrm{C} 34 \mathrm{~S}]$ ratio of 22.5 .

The calculated optical depth range is used to constrain the $\mathrm{CS}(\mathrm{J}=1)$ column density (Equation 9, Goldsmith \& Langer 1999), and with an assumption of local thermodynamic equilibrium (LTE) at a temperature of $10 \mathrm{~K}$, we converted this to total CS column density, $N_{C S}$, (CS column density $\sim 3.5 \times \mathrm{CS}$ $\mathrm{J}=1$ col. dens.). We note that a $50 \%$ error in the assumed temperature of $10 \mathrm{~K}$ would result in a $20-30 \%$ systematic uncertainty in the column density using this LTE method (Maxted et al. 2013).

Assuming an abundance of CS with respect to molecular hydrogen, $[\mathrm{CS}] /\left[\mathrm{H}_{2}\right] \sim 10^{-9}$ (Frerking et al. 1980), a hydrogen column density is estimated, allowing the estimation of $\mathrm{H}_{2}$ mass. We use no beam-filling correction and assume spherical clumps of a size equal to the $7 \mathrm{~mm}$ beam FWHM 
Table 1. The window set-up for the Mopra Spectrometer, MOPS, at $7 \mathrm{~mm}$. The centre frequency, targeted molecular line, targeted frequency, and total mapping noise $\left(\mathrm{T}_{\mathrm{RMS}}\right)$ are displayed.

\begin{tabular}{|c|c|c|c|c|c|}
\hline \multirow{2}{*}{$\begin{array}{l}\text { Centre } \\
\text { frequency } \\
(\mathrm{GHz})\end{array}$} & \multirow{2}{*}{$\begin{array}{l}\text { Molecular } \\
\text { emission line }\end{array}$} & \multirow{2}{*}{$\begin{array}{c}\text { Line } \\
\text { frequency } \\
(\mathrm{GHz})\end{array}$} & \multicolumn{3}{|c|}{$\operatorname{Map} \mathrm{T}_{\mathrm{RMS}}\left(\mathrm{K} \mathrm{ch}^{-1}\right)$} \\
\hline & & & West & North & Northwest \\
\hline 42.310 & ${ }^{30} \mathrm{SiO}(\mathrm{J}=1-0, \mathrm{v}=0)$ & 42.373365 & $\sim 0.07$ & $\sim 0.07$ & $\sim 0.10$ \\
\hline 42.500 & $\mathrm{SiO}(\mathrm{J}=1-0, \mathrm{v}=3)$ & 42.519373 & $\sim 0.07$ & $\sim 0.07$ & $\sim 0.10$ \\
\hline 42.840 & $\begin{array}{l}\mathrm{SiO}(\mathrm{J}=1-0, \mathrm{v}=2) \\
{ }^{29} \mathrm{SiO}(\mathrm{J}=1-0, \mathrm{v}=0)\end{array}$ & $\begin{array}{l}42.820582 \\
42.879922\end{array}$ & $\sim 0.07$ & $\sim 0.08$ & $\sim 0.10$ \\
\hline 43.125 & $\mathrm{SiO}(\mathrm{J}=1-0, \mathrm{v}=1)$ & 43.122079 & $\sim 0.08$ & $\sim 0.07$ & $\sim 0.12$ \\
\hline 43.395 & $\mathrm{SiO}(\mathrm{J}=1-0, \mathrm{v}=0)$ & 43.423864 & $\sim 0.08$ & $\sim 0.09$ & $\sim 0.12$ \\
\hline 44.085 & $\mathrm{CH}_{3} \mathrm{OH}(7(0)-6(1) \mathrm{A}++)$ & 44.069476 & $\sim 0.08$ & $\sim 0.10$ & $\sim 0.12$ \\
\hline 45.125 & $\mathrm{HC}_{7} \mathrm{~N}(\mathrm{~J}=40-39)$ & 45.119064 & $\sim 0.09$ & $\sim 0.12$ & $\sim 0.12$ \\
\hline 45.255 & $\mathrm{HC}_{5} \mathrm{~N}(\mathrm{~J}=17-16)$ & 45.26475 & $\sim 0.09$ & $\sim 0.13$ & $\sim 0.13$ \\
\hline 45.465 & $\mathrm{HC}_{3} \mathrm{~N}(\mathrm{~J}=5-4, \mathrm{~F}=5-4)$ & 45.488839 & $\sim 0.09$ & $\sim 0.13$ & $\sim 0.13$ \\
\hline 46.225 & ${ }^{13} \mathrm{CS}(\mathrm{J}=1-0)$ & 46.24758 & $\sim 0.09$ & $\sim 0.13$ & $\sim 0.13$ \\
\hline 47.945 & $\mathrm{HC}_{5} \mathrm{~N}(\mathrm{~J}=16-15)$ & 47.927275 & $\sim 0.12$ & $\sim 0.15$ & $\sim 0.15$ \\
\hline 48.225 & $C^{34} S(J=1-0)$ & 48.206946 & $\sim 0.12$ & $\sim 0.15$ & $\sim 0.15$ \\
\hline 48.635 & $\operatorname{OCS}(\mathrm{J}=4-3)$ & 48.651604 & $\sim 0.13$ & $\sim 0.15$ & $\sim 0.15$ \\
\hline 48.975 & $\mathrm{CS}(\mathrm{J}=1-0)$ & 48.990957 & $\sim 0.12$ & $\sim 0.16$ & $\sim 0.17$ \\
\hline
\end{tabular}

(radius $\sim 30$ arcsec). Where significant CS(1-0) emission extended beyond an approximate beam-area, sources were divided into segments (east and west in this study) for parameter calculations, and an additional large-scale region was used for an overall mass estimation of the full extent of the CS emission.

\section{RESULTS AND DISCUSSION}

This investigation mapped regions indicated in Figures 1 and 2 in the CS(1-0) transition towards the RX J1713.7-3946 SNR. Significant detections at a velocity consistent with $\mathrm{CO}(1-0,2-1)$-traced cores that are believed to be associated with RX J1713.7-3946 (line-of-sight velocity, $\mathrm{v}_{\mathrm{LSR}}$ $-10 \mathrm{~km} \mathrm{~s}^{-1}$ according to Moriguchi et al.2005; Fukui 2008) were recorded. In addition to this, we found gas consistent with the background Norma arm $\left(\mathrm{v}_{\mathrm{LSR}} \sim-70 \mathrm{~km} \mathrm{~s}^{-1}\right)$ and the $3 \mathrm{kpc}$-expanding arm $\left(\mathrm{v}_{\mathrm{LSR}} \sim-120 \mathrm{~km} \mathrm{~s}^{-1}\right)$. For the latter arm, we also note the detection of the $\mathrm{CH}_{3} \mathrm{OH}(7-6)$ transition.

In addition to our $\mathrm{CS}$ and $\mathrm{CH}_{3} \mathrm{OH}$ detections, transient detections of the $\mathrm{v}=1$ and 2 vibrational modes of the $\mathrm{SiO}(1-$ $0)$ rotational transition were present towards one location $\left(\mathrm{v}_{\mathrm{LSR}} \sim-5 \mathrm{~km} \mathrm{~s}^{-1}\right)$.

\subsection{CS(1-0) Emission}

The CS(1-0) transition has a critical density for emission of $\sim 10^{5} \mathrm{~cm}^{-3}$ (at temperature $\sim 10 \mathrm{~K}$ ) and is ideal for probing the deep, inner regions of molecular clouds. The dense gas traced in this study is displayed in Figures 3, 6, and 7 and are discussed in the following sections. Table 2 is a compilation of spectral line fit parameters and gas parameters, including line-of-sight velocity, line-width, optical depth, $\mathrm{H}_{2}$ column density, and mass. These values are used as a basis for the discussion of dense gas towards RX J1713.7-3946.

\subsubsection{CS(1-0) emission between $v_{\mathrm{LSR}} \sim-12.5$ and $-7.5 \mathrm{~km} \mathrm{~s}^{-1}$}

Figure 3 is an image of CS(1-0) emission between $v_{\text {LSR }}$ $=-12.5$ and $-7.5 \mathrm{~km} \mathrm{~s}^{-1}$, which features detections corresponding to cores believed to be associated with the RX J1713.7-3946 SNR.

The dense components of cores A, B, C, and D were previously studied in detail by us (Maxted et al. 2012a), revealing multiple detections of six species (and several isotopologues), including CS(1-0) emission towards Cores A, $\mathrm{C}$, and $\mathrm{D}$. This allowed the mass of the dense molecular gas to be estimated and these are re-stated (along with our new results) in Table 2.

In our new survey (this work), we revealed CS(1-0) emission towards Cores $\mathrm{G}$ and $\mathrm{L}$, with peak intensities of $\sim 1.5 \mathrm{~T}_{\mathrm{RMS}}$. After integration (between velocities, $\mathrm{v}_{\mathrm{LSR}}=$ -12.5 and $-7.5 \mathrm{~km} \mathrm{~s}^{-1}$ ), Cores $\mathrm{G}$ and $\mathrm{L}$ were detected at a pre-trial level of $\sim 4-4.5 \sigma$. Since the CS(1-0) emission intensities in these two cores are very low, the resultant CS(1-0) optical depth estimates are poorly constrained. However, given that the CS(1-0) intensities of Cores $\mathrm{G}$ and $\mathrm{L}$ are relatively low compared to those of Cores A, C, and D (which have LTE masses in the range $12-120 \mathrm{M}_{\odot}$ ), we favour the optically thin solutions. It follows that dense molecular gas masses of Cores $\mathrm{G}$ and $\mathrm{L}$ are on the order of $\sim 1 \mathrm{M}_{\odot}$, and do not represent a significant proportion of mass in the RX J1713.7-3946 field at $\mathrm{v}_{\mathrm{LSR}} \sim-10 \mathrm{~km} \mathrm{~s}^{-1}(<0.1 \%)$, therefore they probably do not play an important role in the dynamics of the SNR-shock or the production of $\gamma$-ray emission. 
Table 2. Parameters of CS(1-0) emission towards RX J1713.7-3946. Galactic coordinates, [l,b], line-of-sight velocity, $\mathrm{v}_{\text {LSR }}$, assumed distance, line-width (full-width-half-maximum), $\Delta \mathrm{v}_{\mathrm{FWHM}}$, optical depth, average $\mathrm{H}$ column density, $\overline{\mathrm{N}_{\mathrm{H}_{2}}}$, and mass of $\mathrm{H}, M$, are indicated.Statistical uncertainties are on the order of $20 \%$, so errors are likely dominated by the systematics introduced by the analysis assumptions.

\begin{tabular}{|c|c|c|c|c|c|c|c|}
\hline Name $^{a}$ & $\begin{array}{c}\text { Direction } \\
\quad[l, b]\end{array}$ & $\begin{array}{c}\mathrm{v}_{\mathrm{LSR}} \\
\left(\mathrm{km} \mathrm{s}^{-1}\right)\end{array}$ & $\begin{array}{c}\text { Assumed } \\
\text { Distance }(\mathrm{kpc})\end{array}$ & $\begin{array}{l}\Delta \mathrm{v}_{\mathrm{FWHM}} \\
\left(\mathrm{km} \mathrm{s}^{-1}\right)\end{array}$ & $\begin{array}{l}\text { Optical } \\
\text { Depth }^{b}\end{array}$ & $\begin{array}{c}{\overline{N_{H_{2}}}}^{c} \\
\left(\times 10^{21} \mathrm{~cm}^{-2}\right)\end{array}$ & $M\left(\mathrm{M}_{\odot}\right)$ \\
\hline Core $\mathrm{A}^{d}$ & $346.94^{\circ},-0.32^{\circ}$ & $-9.82 \pm 0.02$ & 1.0 & $1.25 \pm 0.06$ & $0-0.44$ & $3-4$ & $12-15$ \\
\hline Core $\mathrm{C}^{d}$ & $347.08^{\circ},-0.40^{\circ}$ & $-11.76 \pm 0.01$ & 1.0 & $2.08 \pm 0.03$ & $2.71 \pm 0.29$ & $55 \pm 4$ & $40 \pm 3$ \\
\hline Core $\mathrm{D}^{d}$ & $347.31^{\circ}, 0.01^{\circ}$ & $-9.1 \pm 0.1$ & 1.0 & $2.5 \pm 0.3$ & $0-4.1$ & $40-170$ & $30-120$ \\
\hline Core G & $347.033^{\circ},-0.067^{\circ}$ & $-11.2 \pm 1.1$ & 1.0 & $0.9 \pm 0.3$ & $0-33^{e}$ & $0.7-400^{e}$ & $1-200^{f}$ \\
\hline Core L & $347.433^{\circ},-0.133^{\circ}$ & $-10.8 \pm 0.4$ & 1.0 & $1.6 \pm 1.4$ & $0-20^{e}$ & $0.1-600^{e}$ & $0.3-300^{f}$ \\
\hline Clump N1-west & $347.18^{\circ},+0.01^{\circ}$ & $-70.1 \pm 0.2$ & 6.0 & $4.6 \pm 0.5$ & $0-4.7$ & $130-610$ & $(2-10) \times 10^{3}$ \\
\hline Clump N1-east & $347.24^{\circ},-0.03^{\circ}$ & $-69.4 \pm 0.4$ & 6.0 & $8.0 \pm 1.3$ & $0-7.5$ & $150-1100$ & $(3-20) \times 10^{3}$ \\
\hline Clump N3 & $347.24^{\circ},+0.02^{\circ}$ & $-76.6 \pm 0.3$ & 6.0 & $5.5 \pm 0.9$ & $0-11$ & $73-800$ & $(1-14) \times 10^{3}$ \\
\hline $\mathrm{N} 1+\mathrm{N} 3$ region & - & - & - & - & - & - & $(5-30) \times 10^{3}$ \\
\hline Clump N2 & $347.00^{\circ},-0.01^{\circ}$ & $-70.9 \pm 0.2$ & 6.0 & $2.4 \pm 0.5$ & $0-8.8$ & $39-340$ & $(7-60) \times 10^{2}$ \\
\hline Clump T1-east & $347.21^{\circ},-0.09^{\circ}$ & $-120.0 \pm 0.2$ & 6.5 & $2.2 \pm 0.3$ & $0-4.4$ & $64-280$ & $(1-6) \times 10^{3}$ \\
\hline Clump T1-west & $347.14^{\circ},-0.07^{\circ}$ & $-119.5 \pm 0.1$ & 6.5 & $1.6 \pm 0.3$ & $0-4.6$ & $45-210$ & $(9-40) \times 10^{2}$ \\
\hline $\mathrm{T} 1$ region & - & - & - & - & - & - & $(3-12) \times 10^{3}$ \\
\hline
\end{tabular}

${ }^{a}$ Name convention from Moriguchi et al. (2005) and this work.

${ }^{b}$ Assuming $[C S] /\left[C^{34} S\right]=22.5$ and using the $\mathrm{C}^{34} \mathrm{~S}(1-0)$-band $\mathrm{T}_{\mathrm{RMS}}$ to estimate upper limits where no $\mathrm{C}^{34} \mathrm{~S}(1-0)$ emission is detected.

${ }^{c}$ Thermal Equilibrium (LTE) assumption at a temperature of $10 \mathrm{~K}$. Averaged over beam size.

${ }^{d}$ Maxted et al. (2012a).

${ }^{e}$ Lower values favoured (see Section 4.1.1).

Indeed, it appears that the total CS(1-0)-derived mass of all the surveyed cores represents only a fraction of the total gas mass in the region. The total $\mathrm{CO}(1-0)$-derived mass of $\sim 1$ $\times 10^{4} \mathrm{M}_{\odot}$ towards RX J1713.7-3946 (Fukui et al. 2012) is significantly larger than the total $\operatorname{CS}(1-0)$-derived mass (dense core) components of mass $80-200 \mathrm{M}_{\odot}$ in our surveyed region (a subset containing the densest molecular gas of the CO-traced region).

Previous authors have noted a small-scale anticorrelation between $\mathrm{CO}$ emission peaks at $\sim 1 \mathrm{kpc}\left(\mathrm{v}_{\mathrm{LSR}} \sim-18\right.$ $0 \mathrm{~km} \mathrm{~s}^{-1}$ ) and X-ray emission (see Section 1), suggesting a $\mathrm{SN}$ shock interaction with gas. Indeed, two X-ray peaks lie on the outskirts of the CS(1-0) (and CO(4-3,7-6), Sano et al. 2010) emission corresponding to Core $C$ (see Figure 4) and a similar X-ray peak lies coincident with Core D. The same cannot be said for Cores A, G, and L, but, although the densest regions of Cores G and A sit outside the RX J1713.7-3946 $\mathrm{X}$-ray boundary, X-ray flux peaks do seem to correspond to regions directly adjacent to the CS(1-0) boundaries. This possibly indicates a shock-compression of the Core $\mathrm{G}$ and A gas, however we have previously noted that these features may alternatively be consistent with photoelectric absorption of X-rays emitted behind the molecular cores (Maxted et al. 2012a).

Previous authors (e.g. Moriguchi et al. 2005) have noted indicators of star formation towards RX J1713.7 -3946, and new data from HOPS $\left(\mathrm{H}_{2} \mathrm{O}\right.$ Plane Survey; Walsh et al. 2011) supports this picture. On examination of the HOPS catalogue, we found narrow $\left(\Delta \mathrm{v}_{\mathrm{FWHM}} \sim 1.1 \mathrm{~km} \mathrm{~s}^{-1}\right) 12 \mathrm{~mm} \mathrm{H}_{2} \mathrm{O}$ maser emission at a velocity and position consistent with Core $\mathrm{D}$ $\left(\mathrm{G} 347.32+0.2, \mathrm{v}_{\mathrm{LSR}} \sim-8.5 \mathrm{~km} \mathrm{~s}^{-1}\right)$. The location of this maser is indicated in Figures 3 and 4.
Gas as CR Target Material:. The gas between $\mathrm{v}_{\mathrm{LSR}}$ $\sim-12.5$ and $-7.5 \mathrm{~km} \mathrm{~s}^{-1}$ may be acting as CR target material in a hadronic gamma-ray emission scenario for RX J1713.7-3946, so we investigate the effect of the mass of CS(1-0)-traced gas.

Aharonian et al. (1991) derived a relation to predict the hadronic flux of $\gamma$-rays above a given energy from the mass of CR-target material, assuming an $E^{-1.6}$ integral power law spectrum. We can calculate the expected $\gamma$-ray flux above energy $E_{\gamma}$,

$$
F\left(\geq E_{\gamma}\right)=2.85 \times 10^{-13} E_{T e V}^{-1.6}\left(\frac{M_{5}}{d_{k p c}^{2}}\right) k_{C R} \mathrm{~cm}^{-2} \mathrm{~s}^{-1}
$$

where $M_{5}$ is the gas mass in units of $10^{5} \mathrm{M}_{\odot}, d_{k p c}$ is the distance in units of kpc, $k_{C R}$ is the CR enhancement factor above that is observed at Earth, $E_{T e V}$ is the lower $\gamma$-ray energy in units of TeV, and $E_{\gamma}=1 \mathrm{TeV}$.

Given that in a hadronic scenario $\gamma$-ray flux is proportional to mass, one can assume that the dense CS(1-0)-traced component ( $\sim$ few per cent of the total mass) may only account for $\sim$ few per cent of the total $\gamma$-ray flux. In a leptondominated scenario, a hadronic component would comprise an even smaller proportion of the total flux (e.g. Zirakashvili \& Aharonian 2010).

Figure 5 is a graph of the predicted hadronic $\gamma$-ray flux above $1 \mathrm{TeV}$ from various gas components (traced using $\mathrm{HI}$, $\mathrm{CO}$, and CS) assumed to be acting as target material for CRs accelerated in the RX J1713.7-3946 shock at a distance of $1 \mathrm{kpc}$ (using Equation 1). It is predicted that the $\mathrm{CR}$ enhancement would have to be as high as $k_{C R} \sim 1000$ for the RX J1713.7-3946 gamma-ray flux above $1 \mathrm{TeV}$, of 


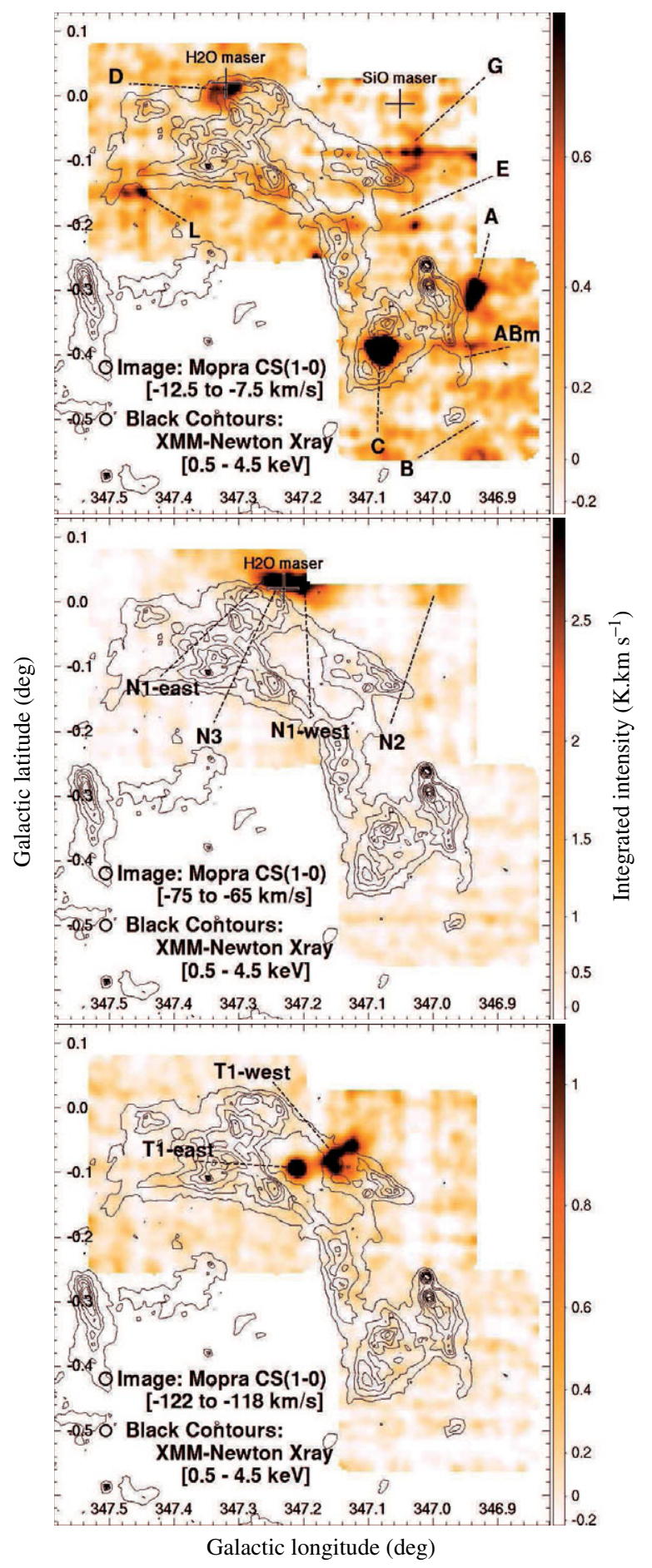

Figure 4. Colour image of integrated CS(1-0) emission, shown for three different ranges indicated in each panel, overlaid with XMM-Newton 0.54.5 keV X-ray contours from Acero et al. (2009). X-ray contours span $0.003-0.015 \mathrm{~cm}^{-2} \mathrm{~s}^{-1}$ in increments of $0.003 \mathrm{~cm}^{-2} \mathrm{~s}^{-1}$. Core names and the position of masers are indicated. The Mopra $7 \mathrm{~mm}$ and XMM-Newton beam FWHM are represented by the small circles next to the text on the picture. $\sim 6 \times 10^{-11} \mathrm{~cm}^{-2} \mathrm{~s}^{-1}$, to be entirely from hadronic processes. In such a scenario, the dense gas components (as traced by CS(1-0) emission) would contribute to a gamma-ray flux of $\sim 3 \times 10^{-13} \mathrm{~cm}^{-2} \mathrm{~s}^{-1}$. This flux may be detectable with the current HESS sensitivity, but the HESS beam FWHM is too large to resolve molecular cores at a scale of $\sim 1 \mathrm{arcmin}$. Future experiments such as the CTA gamma-ray telescope (The CTA Consortium 2010) may reach the arcminute resolution required to do this.

A CR enhancement factor of $k_{C R} \sim 1000$ towards RX J1713.7-3946 is plausible, supported by work by Aharonian \& Atoyan (1996). Figure 1b of this paper displays $\mathrm{CR}$ enhancement factors of the order $k_{C R} \sim 10^{3}$ at a distance of $10 \mathrm{pc}$ from a SNR of age $10^{3} \mathrm{yr}$ (similar to the RX J1713.7-3946 age). The source spectral index in these simulations was 2.2 and the CR diffusion coefficient was $10^{26} \mathrm{~cm}^{2} \mathrm{~s}^{-1}$ (slow diffusion like that seen towards, W28, e.g. Gabici et al. 2010).

We estimate the total cumulative kinetic energy of CRs above an energy of $1 \mathrm{GeV}$ (assuming a pure $\mathrm{E}^{-2.6}$ power law down to $1 \mathrm{GeV}$ ) in the RX J1713.7-3946 region, to be,

$$
\begin{aligned}
E_{C R \text { tot }}= & E_{p} n_{C R} d V d E_{P} \\
& \sim \frac{4}{3} \pi r_{S N R}^{3} \int_{1 \mathrm{GeV}}^{\infty} E_{P} n_{C R} d E_{P} \\
& \sim\left(1.2 \times 10^{59} \mathrm{~cm}^{3}\right)\left(5.6 \times 10^{-11} \mathrm{GeV} \mathrm{cm}^{-3}\right) k_{C R} \\
& \sim k_{C R} \times 10^{46} \mathrm{erg}
\end{aligned}
$$

where $V$ is the SNR volume, $E_{P}$ is CR energy, $n_{C R}$ is the $\mathrm{CR}$ density, and $r_{S N R}$ is the radius of a spherical region of CR enhancement, $k_{C R}$ (assumed to be $10 \mathrm{pc}$, consistent with the RX J1713.7-3946 radius). Assuming a SN blast kinetic energy of $10^{51} \mathrm{erg}$, a CR enhancement of 1000 would correspond to $\sim 1 \%$ of the SN blast kinetic energy being injected into $>1 \mathrm{GeV}$ protons, so energetically, a hadronic scenario is consistent.

The presented CR enhancement factor values were calculated under the assumption that all the gas in the region is taken into account, well traced by the latest $\mathrm{HI}, \mathrm{CO}$, and CS studies (Fukui et al. 2012; Maxted et al. 2012b), which may not be entirely valid. A so-called 'dark' component of gas, where carbon is in atomic/ionic form (not in molecules such as $\mathrm{CO}$ or CS) and hydrogen is in molecular form (therefore does not emit the atomic $\mathrm{H} 21 \mathrm{~cm}$ line) exists and is not taken into account by the tracers considered in studies of RX J1713.7-3946. Wolfire, Hollenbach, \& McKee (2010) predicted that this dark component may comprise $\sim 30 \%$ of the total mass of an average cloud but could be even higher in regions with enhanced CR ionisation rates, as is likely around RX J1713.7-3946.

Our method also required the assumption of an $E^{-1.6}$ integral power law CR spectrum, which is not supported by studies of the RX J1713.7-3946 high energy photon spectrum. The RX J1713.7-3946 $\gamma$-ray spectrum is consistent with an 


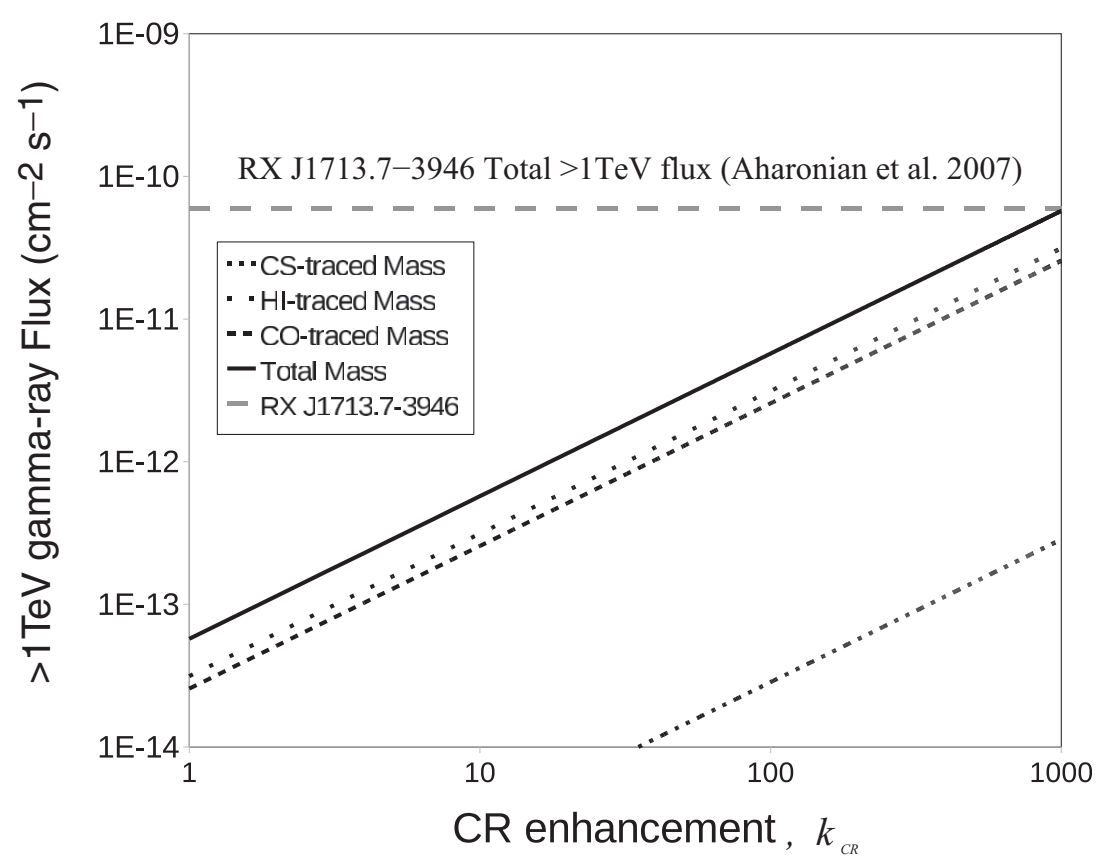

Figure 5. The predicted hadronic $\gamma$-ray flux as a function of CR-enhancement for gas components possibly associated with RX J1713.7-3946 $\left(\mathrm{v}_{\mathrm{LSR}} \sim-10 \mathrm{~km} \mathrm{~s}^{-1}\right)$. The contributions from HI and CO-traced components towards the RX J1713.7-3946 region and CS(1-0) emission from five cores are included (this work). The total predicted hadronic $\gamma$-ray flux remains less than the measured RX J1713.7-3946 $\gamma$-ray flux for CR enhancement values less than 1000 . A distance of $1 \mathrm{kpc}$ was assumed.

$E^{-1.3} \mathrm{CR}$ integral spectrum, reflective of a pure power law $\chi^{2}$-minimisation fit to the $\gamma$-ray spectrum (Aharonian et al. 2007). Using the fact that the total energy injected in CRs bewteen $1 \mathrm{GeV}$ and $1 \mathrm{PeV}$ is expected to be approximately fixed $\left(\sim 10^{50} \mathrm{erg}\right)$, we can pivot the assumed CR energy spectrum about an energy of $\sim 1 \mathrm{TeV}$. This would correspond to a pivot energy of $\sim 0.1 \mathrm{TeV}$ in the resultant hadronic gammaray spectrum. It follows that Equation 1 can be scaled to become $F\left(\geq E_{\gamma}\right) \sim 4.6 \times 10^{-13} E^{-1.3}{ }_{T e V}\left(M_{5} / d^{2}{ }_{k p c}\right) k_{C R}$. The resultant CR enhancement factors (for CR energy $\geq 1 \mathrm{TeV}$ ) presented in this paper would thus reduce by $\sim 30 \%$.

It is clear that in a hadronic scenario, the atomic and molecular mass traced by $\mathrm{HI}$ and $\mathrm{CO}$ would be the dominant contributor to $\gamma$-ray emission. Despite this, tell-tale signs of hadronic emission might feasibly be detected in the form of small-scale hardening and localisation of high-energy spectra correlated with dense gas components. Such phenomena would result from energy-dependent CR diffusion into dense gas in turbulent regions that experience a suppressed diffusion level. This might act to hinder the transport of lower energy CRs more than higher energy CRs and result in a harder than average spectrum (e.g. Gabici et al. 2009; Casanova et al. 2010; Fukui et al. 2012; Maxted et al. 2012a).

In a previous paper (Maxted et al. 2012a), we estimated the level of penetration of CRs of a range of energies $(10 \mathrm{GeV}$ to $10^{2.5} \mathrm{TeV}$ ) into a homogenous molecular region (average density $300 \mathrm{~cm}^{-3}$ ) of radius $0.62 \mathrm{pc}$ (similar to Core C) within a time equal to the RX J1713.7-3946 age ( 1 $600 \mathrm{yr})$. We found that for decreasing values for the diffusion-suppression coefficient, $\chi$ (see Equation), the level of CR penetration into molecular cores may plausibly be decreased, particularly at lower energies.

For a plausible guide on the level of CR penetration into dense gas, we used the 3D random walk distance, $d=\sqrt{6 D t}$, where $t$ is time and the diffusion coefficient, $D$, was parametrised as,

$$
D\left(E_{P}, B\right)=\chi D_{0}\left(\frac{E_{P} / \mathrm{GeV}}{B / 3 \mu \mathrm{G}}\right)^{0.5}\left[\mathrm{~cm}^{2} \mathrm{~s}^{-1}\right],
$$

where $D_{0}$ is the galactic diffusion coefficient, assumed to be $3 \times 10^{27} \mathrm{~cm}^{2} \mathrm{~s}^{-1}$ to fit CR observations (Berezinskii et al. 1990), and $\chi$ is the diffusion suppression coefficient (assumed to be $<1$ inside the core, 1 outside), a parameter invoked to account for possible deviations of the average galactic diffusion coefficient inside molecular clouds (Berezinskii et al. 1990; Gabici, Aharonian, \& Blasi 2007). $E_{P}$ was the proton energy and $B$ was the magnetic field, calculated to be consistent with Crutcher (1999).

We found that for $\chi=10^{-4}$ and $\chi=10^{-5}$, CR protons with energy $<10 \mathrm{TeV}$ were somewhat excluded from the central $\sim 0.2$ and $\sim 0.5 \mathrm{pc}$ radius, respectively. The total level of exclusion, however, is difficult to confidently constrain from such a simplistic approach, so we are currently working on diffusion models that numerically solve the diffusion equation to accurately predict the CR distribution, 

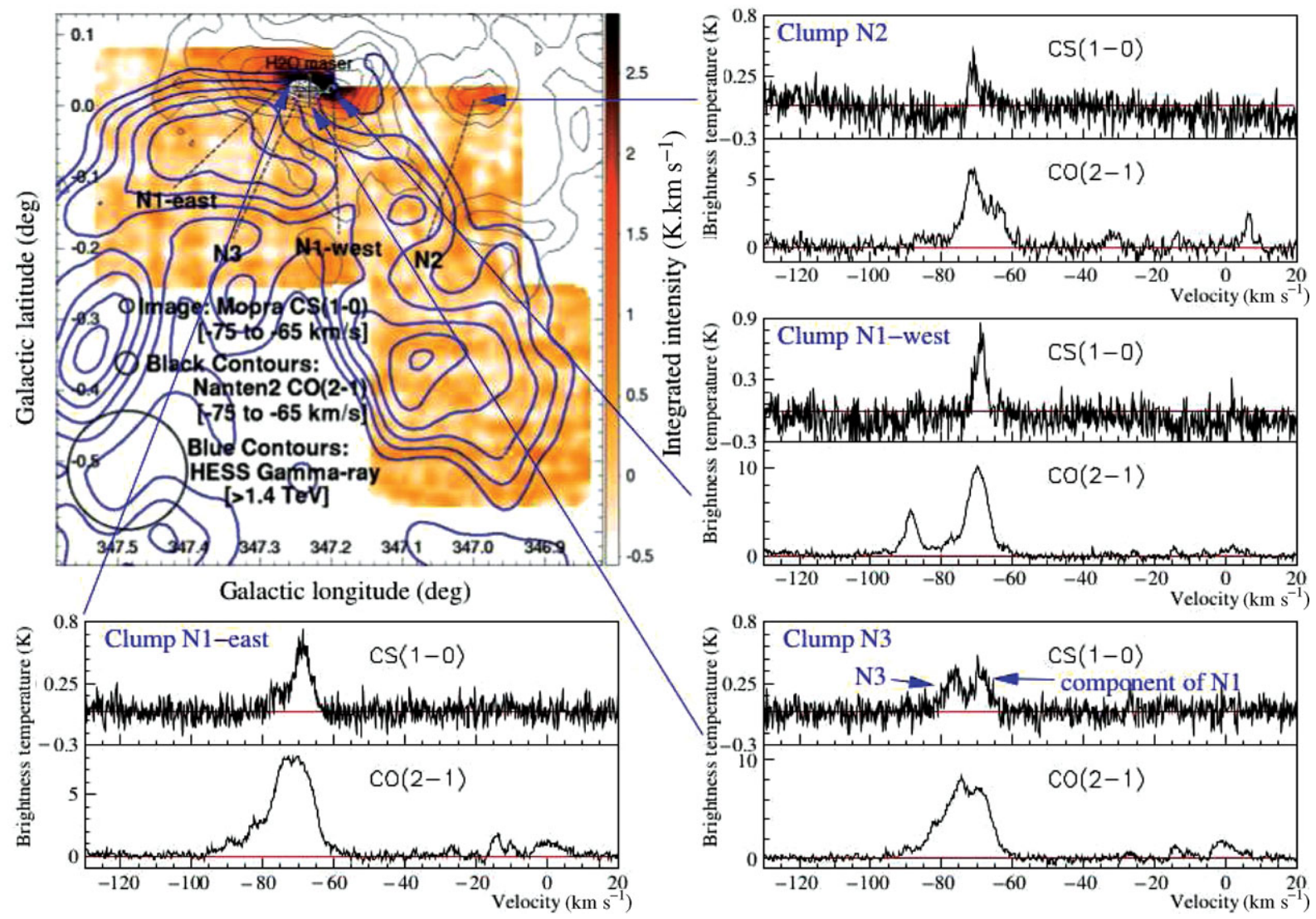

Figure 6. Top left panel: Colour image of integrated CS(1-0) emission $\left(\mathrm{v}_{\mathrm{LSR}}=-75\right.$ to $\left.-65 \mathrm{~km} \mathrm{~s}^{-1}\right)$ from Mopra overlaid with black contours of $\mathrm{CO}(2-1)$ emission ( $\mathrm{v}_{\mathrm{LSR}}=-75$ to $-65 \mathrm{~km} \mathrm{~s}^{-1}$ ) from Nanten2 (Fukui 2008), as well as solid blue contours of HESS $>1.4 \mathrm{TeV}$ excess emission (same levels as for Figure 1). $\mathrm{CO}(2-1)$ emission contour-levels are 10, 20, 30, 40, 50, and $60 \mathrm{~K} \mathrm{~km} \mathrm{~s}^{-1}$. White contours indicate Clump N3 Nanten2 integrated CS(1-0) emission $\left(\mathrm{v}_{\mathrm{LSR}}=-80\right.$ to $\left.-72.5 \mathrm{~km} \mathrm{~s}^{-1}\right)$ with contour-levels of $0.8,1.0,1.2,1.4$, and $1.6 \mathrm{~K} \mathrm{~km} \mathrm{~s}^{-1}$. The position of an $\mathrm{H}_{2} \mathrm{O}$ maser is indicated. Right/Bottom panels: We also show spectral profiles of molecular emission towards locations of interest (indicated).

hence the change in CR enhancement inside dense gas (details to be presented in a future paper). It is expected that diffusion-dependent modelling will yield CR enhancement factors that are functions of not only CR energy, but also gas density, i.e. the $\mathrm{CR}$ enhancement factor, $k_{C R}$, might decrease towards dense clumps, the effect lessening for increasing energy. The result would be harder-than-average hadronic $\gamma$-ray components correlated with dense gas. The prediction of features in $\gamma$-ray spectra will result from future diffusion analyses.

\subsubsection{CS(1-0) emission at other velocities}

In addition to the gas believed to be in association with RX J1713.7-3946 (see Section 4.1.1), dense gas components corresponding to background gas clouds were also detected. Prominent CS(1-0) emission from molecular clouds in the Norma arm and the $3 \mathrm{kpc}$-expanding arm are displayed in Figures 6 and 7, respectively.

On the question of the RX J1713.7-3946 distance (currently thought to be $\sim 1 \mathrm{kpc}$ ), some previous molecular gas surveys favoured an association with gas that featured broad $\mathrm{CO}$ emission components at a distance of $\sim 6.3 \mathrm{kpc}$ $\left(\mathrm{v}_{\mathrm{LSR}} \sim-90 \mathrm{~km} \mathrm{~s}^{-1}\right)$. It was argued that an enhanced $\mathrm{CO}(2-$ 1)/ $\mathrm{CO}(1-0)$ intensity ratio is characteristic of shocked gas (Slane et al. 1999; Butt et al. 2001). The optically subluminous nature of the RX J1713.7-3946 progenitor event (see Section 1) may also be considered to support a larger distance (Fessen et al. 2012). For completeness, we consider these CS(1-0) detections in our analyses.

The Norma Arm $\left(v_{\mathrm{LSR}} \sim-75\right.$ to $\left.-65 \mathrm{~km} \mathrm{~s}^{-1}\right)$ : At a distance of $\sim 6 \mathrm{kpc}$, in the northernmost regions of the surveyed field (Figure 6), are CS(1-0)-traced dense gas components. These components, labelled here as N1, N2, and N3, are within the Norma arm and are coincident with peaks in $\mathrm{CO}(2-$ 1) emission. Due to the extended nature of N1, this region had previously been scrutinised in two sections N1-east and N1-west, with the calculated masses being of the order of a few $\times 10^{3} \mathrm{M}_{\odot}$ for both sides (Maxted et al. 2012b). Further scrutiny of spectral data resulted in the discovery of another coincident dense core component, N3. Clump N3, which is comparable in mass to both N1-east and west $\left(\sim 10^{3} \mathrm{M}_{\odot}\right)$, 


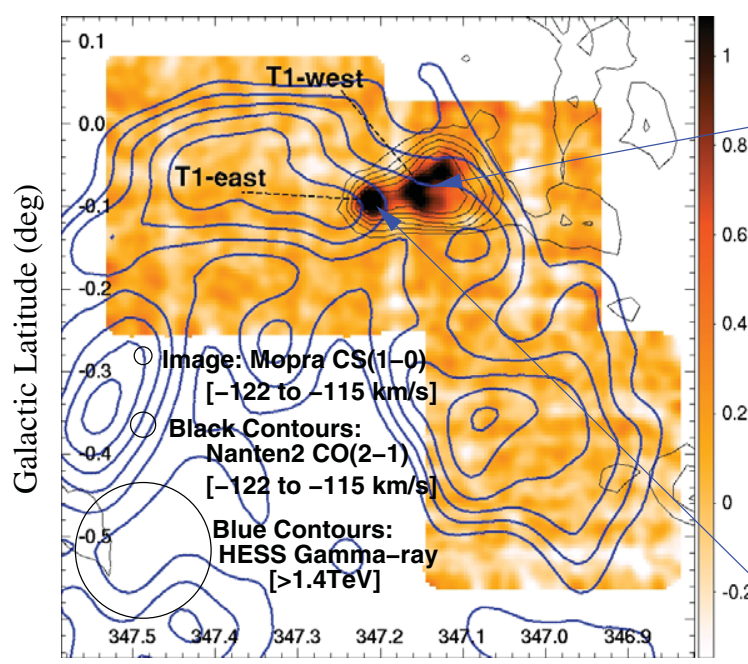

Galactic Longitude (deg)

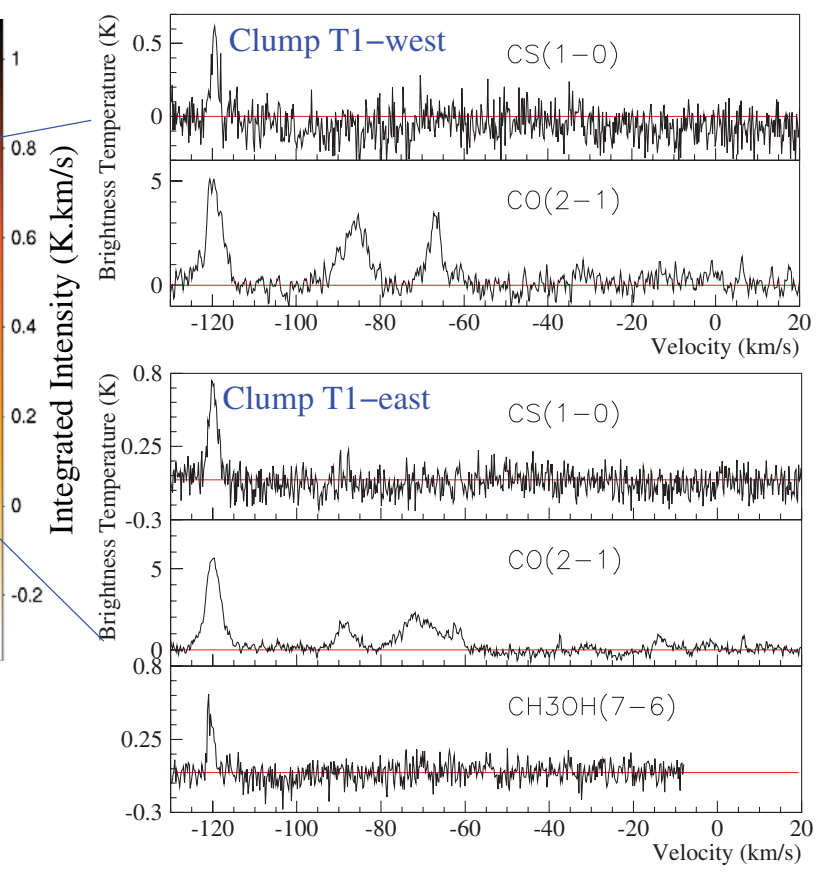

Figure 7. Top left panel: Colour image of integrated CS(1-0) emission $\left(\mathrm{v}_{\mathrm{LSR}}=-122\right.$ to $\left.-115 \mathrm{~km} \mathrm{~s}^{-1}\right)$ from Mopra overlaid with black contours of $\mathrm{CO}(2-1)$ emission $\left(\mathrm{v}_{\mathrm{LSR}}=-122\right.$ to $-115 \mathrm{~km} \mathrm{~s}^{-1}$ ) from Nanten2 (Fukui 2008), as well as solid blue contours of HESS $>1.4 \mathrm{TeV}$ excess emission (same levels as for Figure 1). $\mathrm{CO}(2-1)$ emission contour-levels are 2, 4, 6, 8, 10, and $12 \mathrm{~K} \mathrm{~km} \mathrm{~s}^{-1}$. Right panels: We also show spectral profiles of molecular emission towards locations of interest (indicated).

is apparent as one peak of the double-peaked profile in the bottom-right spectra of Figure 6 (whereas the other peak is an edge of N1), and is shown as white contours in the image. Clump N2, in the north-west of the image in Figure 6, has a mass on the order of $\sim 10^{3} \mathrm{M}_{\odot}$.

Clumps $\mathrm{N} 1$ and $\mathrm{N} 3$ are coincident with the boundary of the RX J1713.7-3946 HESS TeV $\gamma$-ray excess, so might feasibly be CR target material associated with RX J1713.7-3946 if RX J1713.7-3946 is associated with the Norma arm gas (rather than the currently favoured $\mathrm{v}_{\mathrm{LSR}}$ $\sim-10 \mathrm{~km} \mathrm{~s}^{-1} / 1 \mathrm{kpc}$-distance gas), and a hadronic scenario is applicable. In contrast, clump N2 lies outside of the RX J1713.7-3946 TeV $\gamma$-ray boundary, so cannot contribute to the total hadronic $\gamma$-ray flux for any distance solution.

The dense regions of Clumps N1 and N3 lie coincident with the northern boundary of the keV X-ray emission (see Figure 4), which may be consistent with an RX J1713-3946 shock-interaction with Norma-arm gas in the north, but this correspondence may be less convincing than the north-to-east shock-correspondence of the gas at $\mathrm{v}_{\mathrm{LSR}} \sim-10 \mathrm{~km} \mathrm{~s}^{-1}$ (see Section 4.1.1). CS(1-0) spectral lines from Clumps N1 and $\mathrm{N} 3$ are quite broad $\left(\mathrm{v}_{\mathrm{LSR}} \sim 4.5-8 \mathrm{~km} \mathrm{~s}^{-1}\right)$. Such broadening is consistent not only with a shock-gas interaction, but also with multiple components of warm, turbulent gas. The latter scenario is supported by 8 and $24 \mu \mathrm{m}$ data (see Figure 10), which shows several peaks coincident with Clumps N1 and N3. Indeed, we note that narrow $\left(\Delta \mathrm{v}_{\mathrm{FWHM}} \sim 1.2 \mathrm{~km} \mathrm{~s}^{-1}\right) 12 \mathrm{~mm}$
$\mathrm{H}_{2} \mathrm{O}$ maser emission $\left(\mathrm{G} 347.23+0.02, \mathrm{v}_{\mathrm{LSR}} \sim-77.8 \mathrm{~km} \mathrm{~s}^{-1}\right.$; Walsh et al. 2011) is indicative of star formation within the N1 or N3 regions (see Figures 3 and 4), the velocity of the maser being closest to that of the clump N3 (the N3 component has velocity, $\mathrm{v}_{\mathrm{LSR}} \sim-76.6 \pm 0.3 \mathrm{~km} \mathrm{~s}^{-1}$ ).

The 3 kpc-Expanding Arm $\left(v_{\mathrm{LSR}} \sim-122\right.$ to $-118 \mathrm{~km} \mathrm{~s}^{-1}$ ):. Also coincident with the northern boundary of RX J1713-3946 is molecular gas in the $3 \mathrm{kpc}$-expanding arm, at a distance of $\sim 6.5 \mathrm{kpc}$ (Figure 7). The T1 cloud has a mass on the order of $\sim 10^{3} \mathrm{M}_{\odot}$ and contains a distinctive molecular core in the east, T1-east. A hot component in T1east is evidenced by our detection of $\mathrm{CH}_{3} \mathrm{OH}(7-6)$ emission, which is emitted after $\mathrm{CH}_{3} \mathrm{OH}$ is evaporated from dust-grains in hot (Temperature $\sim 100 \mathrm{~K}$ ) conditions (van Dishoek \& Blake 1998). This emission is possibly related to the coincident object, S9 of Churchwell et al. (2006), which appears as an incomplete ring of $8 \mu \mathrm{m}$ emission (see Figure 10). The authors argue that such infrared 'bubbles' result from hot young stars in massive star formation regions. Our $7 \mathrm{~mm}$ results are consistent with T1-east being a hot star-forming core.

Background Gas as CR Target Material:- In Figure 8, we present the predicted hadronic $\gamma$-ray components from dense gas at distances near $\sim 6 \mathrm{kpc}(\mathrm{T} 1, \mathrm{~N} 1$, and N3), for the scenario where this gas is associated with RX J1713.7-3946.

The dense gas components in the Norma and $3 \mathrm{kpc}-$ expanding arms would contribute hadronic $\gamma$-ray emission 

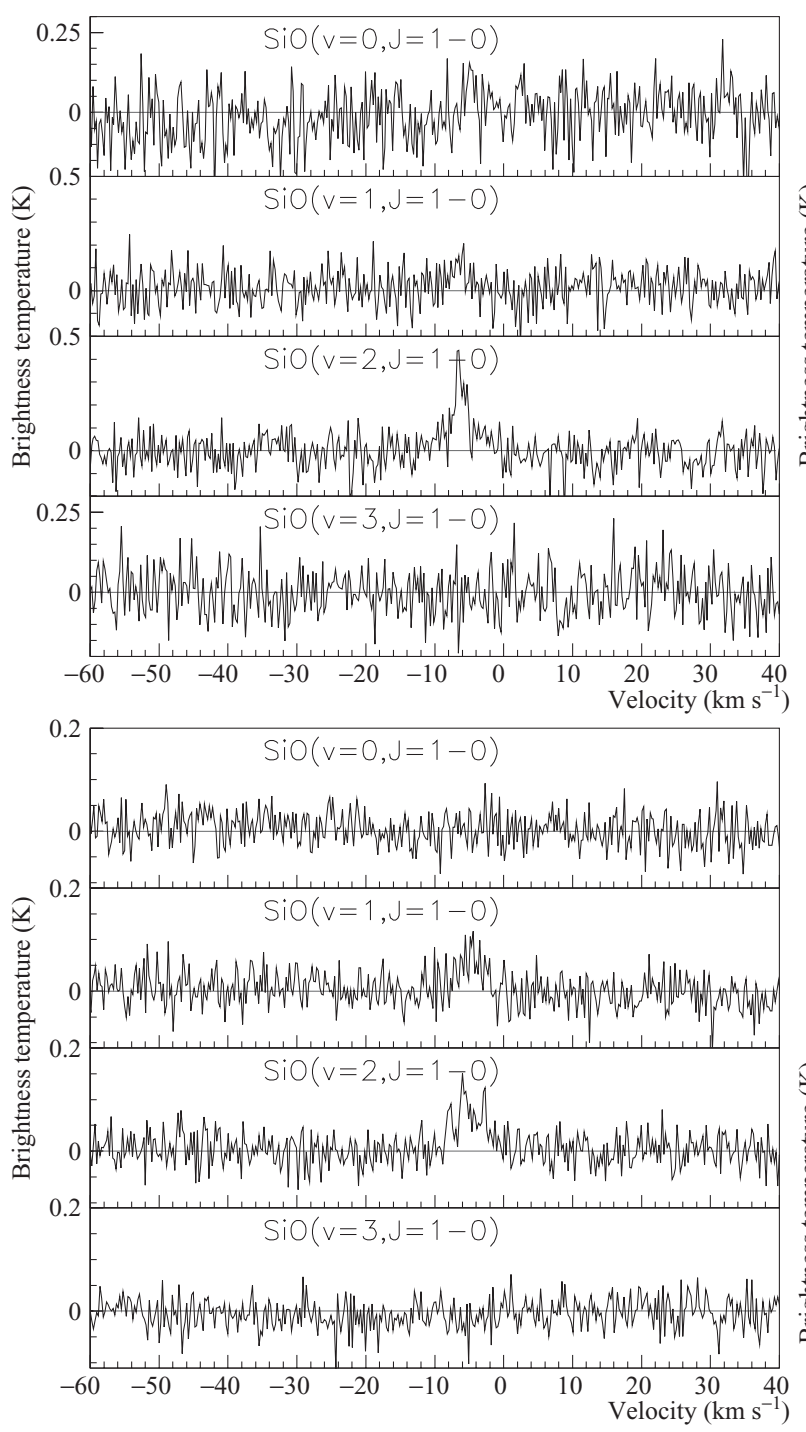

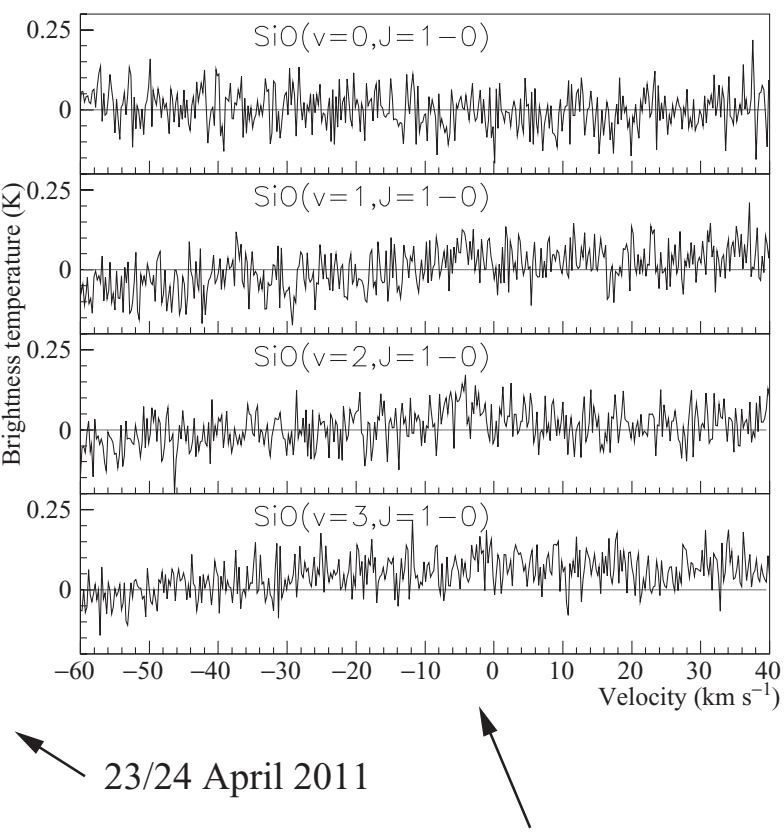

8 April 2012

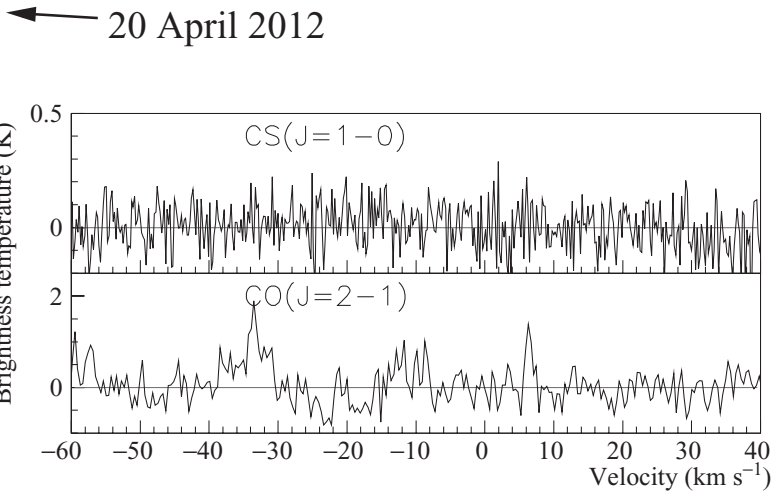

Figure 8. The predicted hadronic $\gamma$-ray flux as a function of CR-enhancement if dense gas currently believed to be background to RX J1713.7-3946 $\left(\mathrm{v}_{\mathrm{LSR}} \sim-70,-120 \mathrm{~km} \mathrm{~s}^{-1}\right)$ were, in fact, associated with RX J1713.7-3946. Note that we only include contributions from CS(1-0) emission. Distances of $6 \mathrm{kpc}$ and $6.5 \mathrm{kpc}$ were assumed for N1/3 and T1, respectively.

at a comparable level to that of the dense gas towards the $\sim 1 \mathrm{kpc}$ region for a given CR enhancement factor, so expected CR enhancement estimates are not likely to help in favouring a specific distance solution. Moriguchi et al. (2005) systematically compared the angular correlation between the RX J1713.7-3946 X-ray emission and CO(1-0) emission at a wide range of line of sight velocities, and concluded that gas in the $\sim 1 \mathrm{kpc}$ region had better angular correspondence than other line-of-sight gas components (including the Norma and $3 \mathrm{kpc}$-expanding arm gas), thus favouring the $\sim 1 \mathrm{kpc}$ distance for RX J1713.7-3946. In a hadronic scenario, correlation is expected between gas and $\gamma$-ray emission, so their argument can be extended to include the hadronic production of gamma-rays, in which case gamma-ray correspondence favours the $\sim 1 \mathrm{kpc}$ distance. Modelling by Inoue et al. (2012) found that the large-scale correlation between $\mathrm{CO}(1-0)$ and the HESS TeV $\gamma$-ray emission supported the $\sim 1 \mathrm{kpc}$ gas association, a model also supported by a small-scale gas-keV $\mathrm{X}$-ray anticorrelation.

We note that the coincident dense gas components of these galactic arms, despite being significantly more massive than the $\sim 1 \mathrm{kpc}$ gas, would produce a flux of $\gamma$-rays $(\sim 4 \times$ $10^{-13} \mathrm{~cm}^{-2} \mathrm{~s}^{-1}$ above $1 \mathrm{TeV}$ ) similar to the dense gas believed to be associated with RX J1713.7-3946, due to the greater distance involved.

We further note that $\mathrm{CO}(2-1)$ spectra towards N1-west and T1 (Figures 6 and 7, respectively) include a component at $\mathrm{v}_{\mathrm{LSR}} \sim-85 \mathrm{~km} \mathrm{~s}^{-1}$, which does not appear to include a detectable dense gas component traced by CS(1-0) emission (above $\mathrm{T}_{\mathrm{RMS}} \sim 0.16 \mathrm{~K} \mathrm{ch}^{-1}$ ). This gas may correspond to 'Cloud A' (Slane et al. 1999; Butt et al. 2001) or gas traced by Moriguchi et al. (2005). 
Table 3 Line parameters for $\mathrm{SiO}$ transitions towards the detected $\mathrm{SiO}$ maser at $[l, b] \sim[347.05,-0.012]$ for three observation periods (location indicated on Figure 3). Background noise, $\mathrm{T}_{\mathrm{RMS}}$, is shown. Velocity of peak, $\mathrm{v}_{\mathrm{LSR}}$, peak intensity, $\mathrm{T}_{\text {Peak }}$, and FWHM, $\Delta \mathrm{v}_{\mathrm{FWHM}}$, were found by fitting Gaussians before deconvolving with the MOPS velocity resolution.

\begin{tabular}{lcclll}
\hline \hline Date & $\begin{array}{c}\mathrm{SiO}(1-0) \\
\text { transition }\end{array}$ & $\begin{array}{c}\mathrm{T}_{\mathrm{RMS}} \\
\left(\mathrm{K} \mathrm{ch}^{-1}\right)\end{array}$ & $\begin{array}{c}\mathrm{v}_{\text {LSR }} \\
\left(\mathrm{km} \mathrm{s}^{-1}\right)\end{array}$ & \multicolumn{1}{c}{$\mathrm{T}_{\text {Peak }}$} & \multicolumn{1}{c}{$\begin{array}{c}\Delta \mathrm{v}_{\mathrm{FWHM}} \\
\left(\mathrm{km} \mathrm{s}^{-1}\right)\end{array}$} \\
\hline \multirow{3}{*}{2011} & $\mathrm{v}=0$ & 0.09 & $b_{-}-4.9 \pm 0.3$ & ${ }^{b} 0.14 \pm 0.05$ & ${ }^{b} 1.5 \pm 0.7$ \\
April 23/24 & $\mathrm{v}=1$ & 0.07 & $-6.5 \pm 0.4$ & $0.11 \pm 0.03$ & $2.7 \pm 1.0$ \\
& $\mathrm{v}=2$ & 0.08 & $-6.3 \pm 0.1$ & $0.33 \pm 0.05$ & $2.6 \pm 0.7$ \\
& $\mathrm{v}=3$ & 0.07 & - & - & - \\
2012 & $\mathrm{v}=0$ & 0.11 & - & - & - ins- \\
April 8 & $\mathrm{v}=1$ & 0.08 & $a_{-}-4.2 \pm 0.3$ & ${ }^{a} 0.08 \pm 0.02$ & ${ }^{a} 3.9 \pm 2.5$ \\
& $\mathrm{v}=2$ & 0.07 & $a_{-}-4.7 \pm 0.6$ & ${ }^{a} 0.10 \pm 0.02$ & ${ }^{a} 5.2 \pm 0.8$ \\
& $\mathrm{v}=3$ & 0.06 & - & - & - \\
2012 & $\mathrm{v}=0$ & 0.03 & - & - & - \\
April 20 & $\mathrm{v}=1$ & 0.03 & $-4.8 \pm 0.3$ & $0.07 \pm 0.01$ & $3.9 \pm 0.6$ \\
& $\mathrm{v}=2$ & 0.03 & $-5.0 \pm 0.3$ & $0.083 \pm 0.009$ & $5.2 \pm 0.6$ \\
\hline \hline
\end{tabular}

${ }^{a}$ Spectral lines difficult to fit, so line-widths were fixed to be equal to those of the 2012 April 20 observations.

${ }^{b}$ Line deemed insignificant in post-trial analysis (see Section 4.2.1)

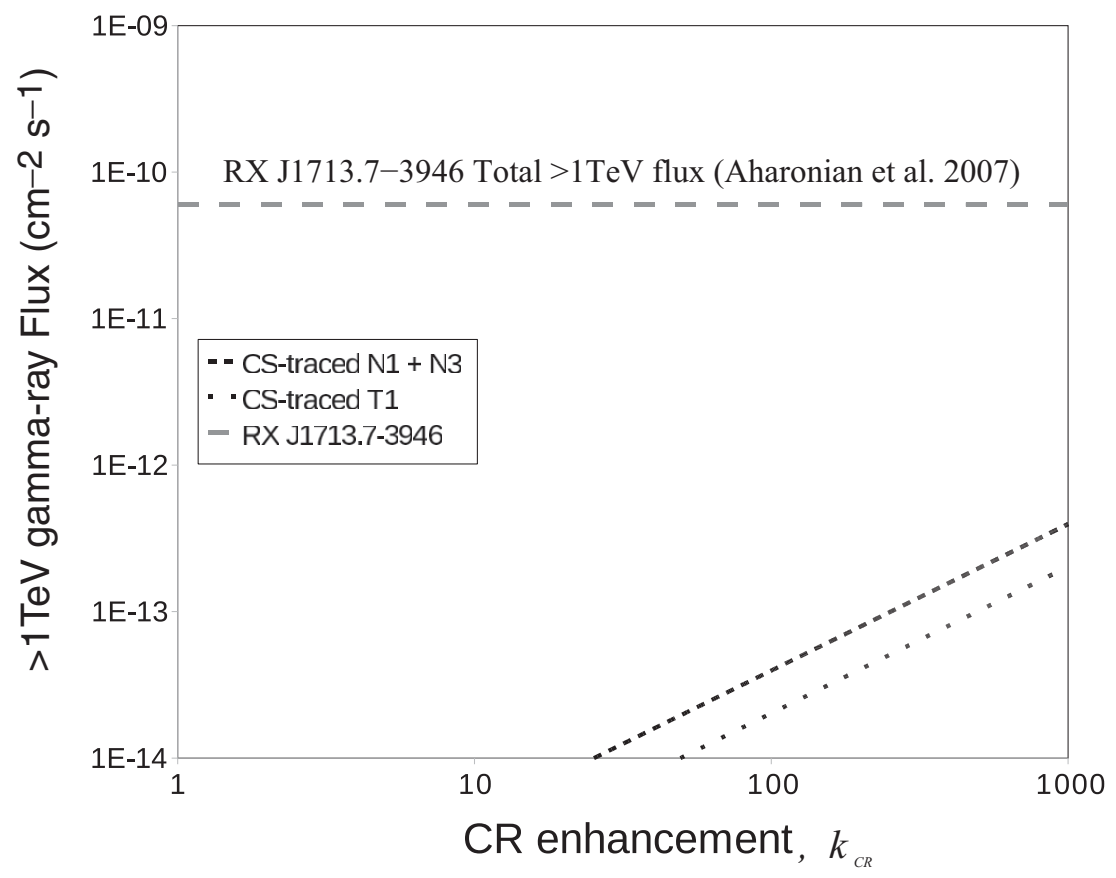

Figure 9. Spectra (Mopra and Nanten2) towards the $\mathrm{SiO}$ maser discovered at $(l, b) \sim(347.05$, -0.012). Data from 2011 April 23/24 were taken from OTF maps. Data from 2012 April are from follow-up pointing data.

\section{2 $\mathrm{SiO}(1-0)$ emission}

A detection of vibrational modes $(\mathrm{v}=1,2)$ of the $\mathrm{SiO}(1-0)$ transition towards $[l, b] \sim[347.05,-0.012]$ in 2011 April prompted follow-up observations that were carried out $1 \mathrm{yr}$ later. The position of this detection is indicated in Figure 3 and spectra are displayed in Figure 9. Spectral characteristics from $\chi^{2}$-minimisation fits are presented in Table 3.

\subsubsection{April}

We measured $\mathrm{SiO}(\mathrm{J}=1-0, \mathrm{v}=2)$ emission that had an intensity greater than the intensity of the lower vibrational mode, $\mathrm{v}=1$, indicating the possible presence of an $\mathrm{SiO}$ populationinversion; thus the emission can be considered as a weak $\mathrm{SiO}$ maser. The $\mathrm{v}=1$ and $\mathrm{v}=2 \mathrm{SiO}(\mathrm{J}=1-0)$ transitions are at consistent velocities, but if a tentative $\mathrm{SiO}(\mathrm{J}=1-0$, $\mathrm{v}=0$ ) detection is real, there is a discrepancy in rest velocity 
(offset from the $\mathrm{v}=1$ and 2 modes by $1.6 \pm 0.14 \mathrm{~km} \mathrm{~s}^{-1}$ and $1.4 \pm 0.1 \mathrm{~km} \mathrm{~s}^{-1}$, respectively). This, in fact, may have been a thermal component, unrelated to the $\mathrm{SiO}$ maser, but since this weak signal was only considered (and searched for) after the detection of the relatively prominent $\mathrm{SiO}(\mathrm{J}=$ $1-0, v=2)$ emission, we estimate the likelihood that it was noise. A naive post-trial analysis that considers the $\mathrm{v}_{\mathrm{LSR}}$ range $\left(9 \mathrm{~km} \mathrm{~s}^{-1}\right)$ in the line-fitting process and the fitted line-width $\left(1.5 \mathrm{~km} \mathrm{~s}^{-1}\right)$ leads to an estimate of six trials. This decreases the significance from $\sim 1.5 \mathrm{~T}_{\mathrm{RMS}}$ to an insignificant $\sim 0.65 \mathrm{~T}_{\mathrm{RMS}}(1.5 / \sqrt{6})$. It follows that although a weak thermal $\mathrm{SiO}$ emission line may exist towards this location, we dismiss the $2011 \mathrm{SiO}(\mathrm{J}=1-0, \mathrm{v}=0)$ detection displayed in Table 3.

We do not dismiss the $2011 \mathrm{SiO}(\mathrm{J}=1-0, \mathrm{v}=1)$ detection using a similar argument since the chance probability of fitting a $\mathrm{v}_{\text {LSR }}$ consistent (within $\sim 0.5 \sigma \sim \pm 0.2 \mathrm{~km} \mathrm{~s}^{-1}$ ) with the original $\mathrm{SiO}(\mathrm{J}=1-0, \mathrm{v}=2)$ detection is approximately $\sim 5 \%$ (trials in a $9 \mathrm{~km} \mathrm{~s}^{-1} \mathrm{v}_{\mathrm{LSR}^{-}}$-range).

\subsubsection{April}

The deep $7 \mathrm{~mm}$ observations of 2012 April 20, allowed a $\sim 2.5 \times$ reduction of spectral noise, and the previously tentative (and insignificant after post-trial analyses) $\mathrm{SiO}(1-0, \mathrm{v}=$ 0 ) line was now absent. Interestingly, the peak intensities of the $\mathrm{v}=1$ and $\mathrm{v}=2 \mathrm{SiO}(\mathrm{J}=1-0)$ emission lines decreased by $\sim 35 \%$ and $\sim 75 \%$, respectively, while the line-width increased by $\sim 45 \%$ and $\sim 100 \%$, respectively.

When we consider the changes in integrated intensity (proportional to the changes in $\mathrm{T}_{\text {Peak }} \times \Delta \mathrm{v}_{\mathrm{FWHM}}$ ), the variation may seem less dramatic and possibly partly due to a change in the velocity distribution of emitting $\mathrm{SiO}$ molecules. The $\mathrm{v}=1$ and $\mathrm{v}=2 \mathrm{SiO}(\mathrm{J}=1-0)$ integrated intensities decrease by $\sim 10 \%$ and $\sim 50 \%$, respectively. Additionally, the rest velocity was observed to change by $\sim 1.5 \mathrm{~km} \mathrm{~s}^{-1}$.

We note that $\chi^{2}$-minimisation Gaussian-fits for the April $8 \mathrm{SiO}(1-0)$ observations were divergent, so the full width half maximum was set manually in order to achieve a $\chi^{2}$ minimisation fit for the other line parameters.

\subsubsection{The SiO Maser Origin}

$\mathrm{SiO}$ masers of this type are commonly associated with evolved stars, or, in very rare cases, star-forming molecular cores. As can be seen from Figure 9, no counterpart is detected in $\mathrm{CO}(2-1)$ or $\mathrm{CS}(1-0)$ bands towards the $\mathrm{SiO}$ maser discovered in this investigation, discrediting the molecular core scenario.

Towards the SiO maser, one midcourse space experiment (MSX) source (G347.0519-00.0100), 20 2MASS catalogue sources, and 31 Spitzer-Glimpse catalogue sources are present within a $30 \operatorname{arcsec}^{\text {radius }}{ }^{2}$, so this maser may have an infrared counterpart (24, 8, and $5.8 \mu \mathrm{m}$; Figures 10 and 11), but a more thorough investigation of this source is beyond the scope of this paper.
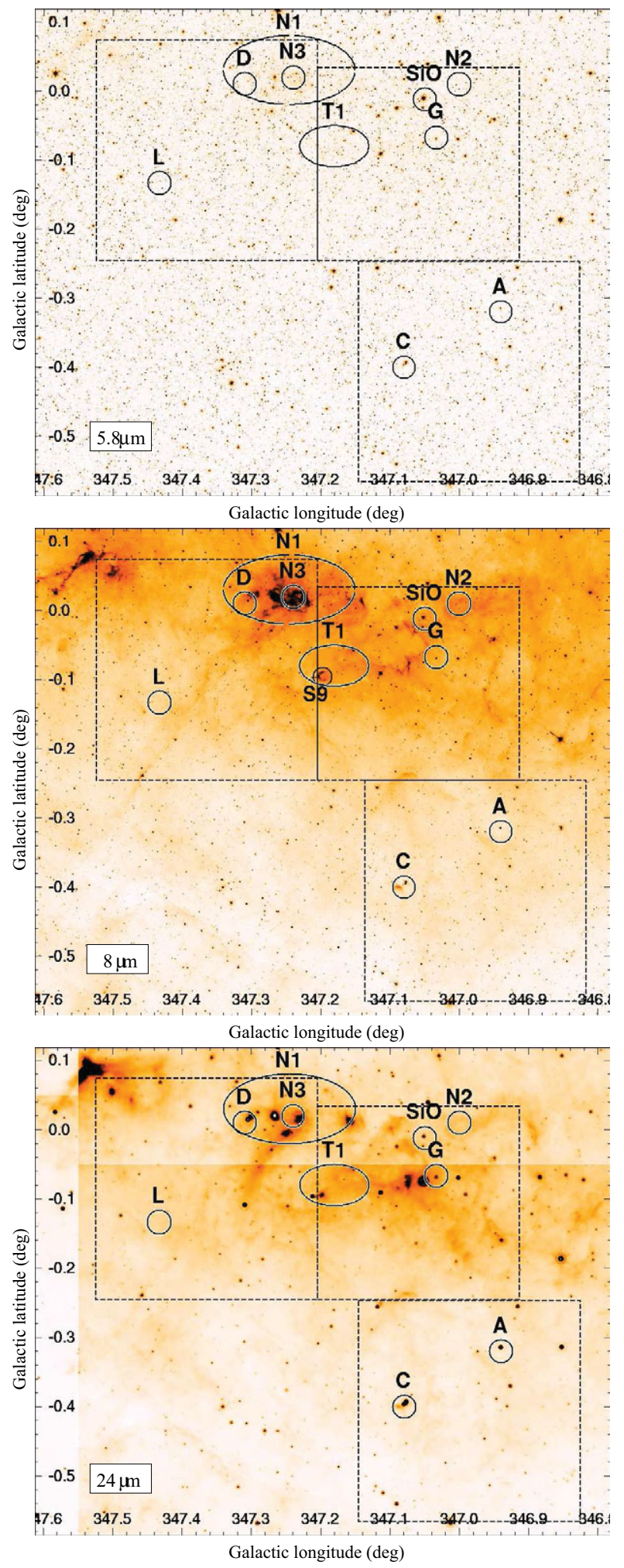

Figure 10. Spitzer $5.8 \mu \mathrm{m}$ (top), $8 \mu \mathrm{m}$ (middle), and $24 \mu \mathrm{m}$ (bottom) emission images. Objects of interest to this investigation (see Section 4.1) are indicated by circles and ellipses. Object $S 9$ towards $T 1$ (see text) is indicated in the middle picture $(8 \mu \mathrm{m})$. Dashed squares indicate regions mapped in $7 \mathrm{~mm}$ wavelengths by Mopra.

\footnotetext{
${ }^{2}$ http://irsa.ipac.caltech.edu/
} 


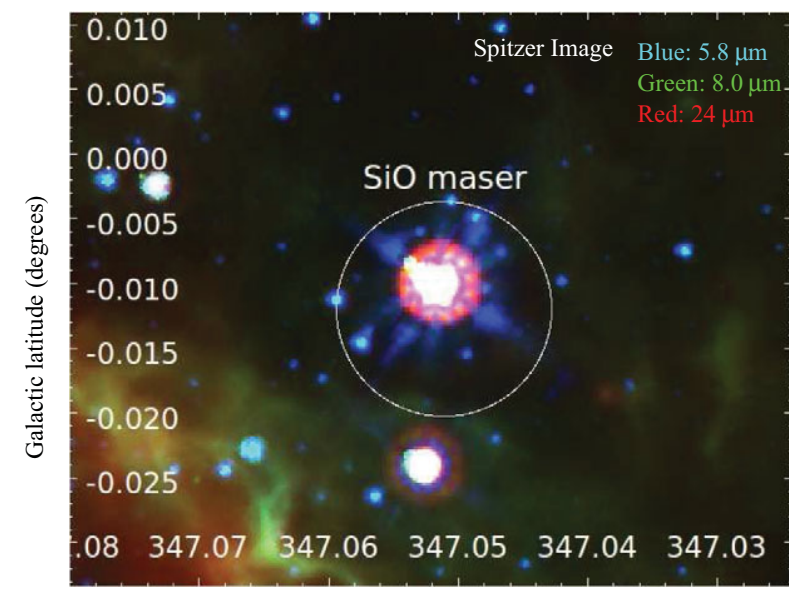

Galactic longitude (degrees)

Figure 11. Three colour image of Spitzer $5.8 \mu \mathrm{m}, 8 \mu \mathrm{m}$, and $24 \mu \mathrm{m}$ emission towards the $\mathrm{SiO}$ maser discovered in our survey. The Mopra $7 \mathrm{~mm}$ beam FWHM is displayed (white circle).

\section{SUMMARY AND CONCLUSION}

We carried out a $7 \mathrm{~mm}$ survey of the northern and western regions of RX J1713.7-3946, targeting the dense gas-tracing CS(1-0) transition and the shock-tracing $\mathrm{SiO}(1-0)$ transition. We report:

(1) the discovery of dense components corresponding to Cores $\mathrm{G}$ and $\mathrm{L}$ at velocity $\mathrm{v}_{\mathrm{LSR}} \sim-11 \mathrm{~km} \mathrm{~s}^{-1}$, as well as gas in the Norma arm at $\mathrm{v}_{\mathrm{LSR}} \sim-70 \mathrm{~km} \mathrm{~s}^{-1}$ and the $3 \mathrm{kpc}$-expanding arm at $\mathrm{v}_{\mathrm{LSR}} \sim-120 \mathrm{~km} \mathrm{~s}^{-1}$;

(2) the discovery of a transient $\mathrm{SiO}(1-0)$ maser, in $\mathrm{v}=1$ and 2 states, outside the western shell of the SNR, possibly generated by an evolved star;

(3) the detection of dense gas and hot gas as indicated by $\mathrm{CS}(1-0)$ and $\mathrm{CH}_{3} \mathrm{OH}(7-6)$-emission coincident with the infrared bubble S9 of Churchwell et al. (2006); and

(4) that a hadronic scenario for the RX J1713.7-3946 SNR would require a CR enhancement above $1 \mathrm{TeV}$ of $\sim 1000$, with dense CS(1-0)-traced components only contributing a gamma-ray flux above $1 \mathrm{TeV}$ of $\sim 3 \times$ $10^{-13} \mathrm{~cm}^{-2} \mathrm{~s}^{-1}$, a few per cent of the total hadronic flux. Systematic effects relating to the proportion of 'hidden' gas and the assumed local CR spectrum may be $\sim 40 \%$ and $\sim 40 \%$, respectively.

\section{ACKNOWLEDGEMENTS}

This work was supported by an Australian Research Council grant (DP0662810, DP1096533). The Mopra Telescope is part of the Australia Telescope and is funded by the Commonwealth of Australia for operation as a National Facility managed by the CSIRO. The University of New South Wales Mopra Spectrometer Digital Filter Bank used for these Mopra observations was provided with support from the Australian Research Council, together with the
University of New South Wales, University of Sydney, Monash University, and the CSIRO.

\section{REFERENCES}

Abdo, A., et al. (Fermi Collab.) 2011, ApJ, 734, 28

Acero, F., Ballet, J., Decourchelle, A., Lemoine-Goumard, M., Ortega, M., Giacani, E., Dubner, G., \& Cassam-Chenaï, G. 2009, A\&A, 505, 157

Aharonian, F. 1991, Ap\&SS, 180, 305A

Aharonian, F., \& Atoyan, A. 1996, ApJ, 309, 917

Aharonian, F., et al. (HESS Collab.) 2006, A\&A, 449, 223

Aharonian, F., et al. (HESS Collab.) 2007, A\&A, 464, 235

Berezhko, E., \& Volk, H. 2010, A\&A, 511, A34

Berezinskii, V., Bulanov, S., Dogiel, V., Ginzburg, V., \& Ptuskin, V. 1990, Astrophysics of Cosmic Rays (Amsterdam: NorthHolland)

Butt, Y., Torres, D., Combi, J., Dame, T., \& Romero, G. 2001, ApJ, 562, L167

Casanova, S., et al. 2010, PASJ, 62, 1127

Cassam-Chenai, G., Decourchelle, A., Ballet, J., Sauvageot, j.-1., Dubner, S., \& Giacani, E. 2004, A\&A, 427, 199

Churchwell, E., et al. 2006, ApJ, 649, 759

Crutcher, R. 1999, ApJ, 520, 706

The CTA Consortium, 2010, arXiv:1008.3703

Ellison, D., Patnaude, D., Slane, P., \& Raymond, J. 2010, ApJ, 712, 287

Fessen, R., Kremer, R., Patnaude, D., \& Milisavljevic, D. 2012, ApJ, 143, 27

Frerking, M., Wilson, R., Linke, R., \& Wannier, P. 1980, ApJ, 240, 65

Fukui, Y. 2008, AIP Conf Proc, 1085, 104

Fukui, Y., Sano, H., Sato, J., Horachi, H., Torii, K., McClureGriffiths, N., Rowell, G., \& Aharonian, F. 2012, ApJ, 746, 82

Fukui, Y., et al. 2003, PASJ, 55, L61

Gabici, S., Aharonian, F., \& Blasi, P. 2007, Ap\&SS, 309, 365

Gabici, S., Aharonian, F., \& Casanova, S. 2009, MNRAS, 396, 1629

Gabici, S., Casanova, S., Aharonian, F., \& Rowell, G. 2010, in Boissier, S., Heydari-Malayeri, M., Samadi, R., VallsGaband, D., eds, Proc. French Soc. Astron. Astrophys., SF2A, 313

Goldsmith, P., \& Langer, W. 1999, ApJ, 517, 209

Inoue, T., Yamazaki, R., Inutsuka, S., \& Fukui, Y. 2012, ApJ, 744, 71

Maxted, N., Rowell, G., Dawson, B., Burton, M., Nicholas, B., Fukui, Y., Walsh, A., Kawamura, A., et al. 2012, MNRAS, 422, 2230

Maxted, N., Rowell, G., Dawson, B., Burton, M., Kawamura, A., Walsh, A., Sano, H., \& Lazendic, J. 2012b, in AIP Conf. Proc., Vol. 1505, High Energy Gamma-Ray Astronomy, eds. F. Aharonian et al. (New York: American Institute of Physics) 253

Maxted, N., Rowell, G., Dawson, B., Burton, M., Fukui, Y., Walsh, A., Kawamura, A., Hirotaka, H., et al. 2013, MNRAS, 434, 2188

Moriguchi, Y., Tamura, K., Tawara, Y., Sasago, H., Yamaoka, T., Onishi, T., \& Fukui, Y. 2005, ApJ, 631, 947 
Nicholas, B., Rowell, G., Burton, M., Walsh, A., Fukui, Y., Kawamura, A., \& Maxted, N. 2012, MNRAS, 419, 251

Pfeffermann, E., \& Aschenbach, B. 1996, MPE, 263, 267

Porter, T., Moskalenko, I., \& Strong, A. 2006, ApJ, 648, L29

Sano, H., et al. 2010, ApJ, 724, 59

Slane, P., Gaensler, B., Dame, T., Hughes, J., Plucinsky, P., \& Green, A. 1999, ApJ, 525, 357

Urquhart, J., et al. 2010, PASA, 27, 321 van Dishoeck, E., \& Blake, G. 1998, A\&A, 36, 317

Walsh, A., Breen, S., Britton, T., Brooks, K., Burton, M., Cunningham, M., Green, J., \& Harvey-Smith, L. 2011, MNRAS, 416,1764

Wang, Z., Qu, Q., \& Chen, Y. 1997, A\&A, 318, L59-L61

Wolfire, M., Hollenbach, D., \& McKee, C. 2010, ApJ, 716, 1191

Zirakashvili, V., \& Aharonian, F. 2010, ApJ, 708, 965

Ziurys, L., Snell, R., \& Dickman, R. 1989, ApJ, 341, 857 